Supporting Information for

\title{
Higly Enantioselective Oxidation of Sulfides to Sulfoxides by a New Oxaziridinium Salt
}

\author{
R. E. del Río, B. Wang, S. Achab, L. Bohé* \\ Institut de Chimie des Substances Naturelles, CNRS \\ Avenue de la Terrasse, 91198 Gif sur Yvette (France) \\ bohe@icsn.cnrs-gif.fr
}

\section{General Information.}

TLC was performed with silicagel plates and visualized either by UV detection or by spraying with phosphomolybdic acid (5\% in ethanol). Column chromatography was performed using silicagel Merck 60 (230-400 mesh) or aluminium oxide Merck 90 active, neutral (70-230 mesh). Melting points were measured using a Leitz-Wetzler apparatus and are uncorrected. Optical rotations were determined at room temperature. NMR spectra were recorded in $\mathrm{CDCl}_{3}$ and chemical shifts $(\delta)$ are reported in parts per million (ppm). Mass spectra and high resolution mass spectra (HRMS) were recorded using electrospray ionization (ESI). Elemental analysis was performed at the microanalytical laboratory of CNRS. The enantiomeric excesses were determined by HPLC using a CHIRALCEL OD column.

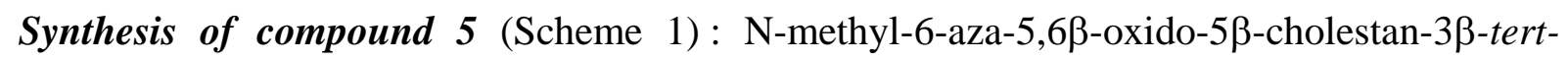
butyldiphenylsilyloxy tetrafluoroborate.

TBDPSCl, (5.2 ml, $2 \mathrm{mmol}$ ) was added, dropwise, to a mixture of cholesterol 1 (5 g, 1.3 mmol) and imidazole (2.64 g, $3.8 \mathrm{mmol}$ ) in dry DMF (8 ml), under stirring at $50^{\circ} \mathrm{C}$. After the addition of the reagent the temperature was raised to $70^{\circ} \mathrm{C}$ and the reaction mixture was stirred for $2 \mathrm{~h}$. It was then cooled to room temperature, poured onto ice and extracted with dichloromethane. The organic layer was washed with water, and dried over sodium sulfate. The solvent was evaporated under vacuum. Chromatography on silicagel, eluting with petroleum ether afforded $7.6 \mathrm{~g}(95 \%)$ of compound 1a; $[\alpha]^{25}-35.89$ (c 1, $\left.\mathrm{CHCl}_{3}\right)$; ${ }^{1} \mathrm{H}$ NMR (300 MHz, $\left.\mathrm{CDCl}_{3}\right) \delta 7.67$ (m, 4H), 7.37 (m, 6H), 5.12 (br d, J = $\left.5.2 \mathrm{~Hz}, 1 \mathrm{H}\right), 3.53$ (m, 1H), 
2.33 (m, 1H), 2.13 (ddd, $\mathrm{J}=$ 13.3, $\mathrm{J}=$ 4.9, J = 1.9 Hz, 1H), 1.98 (m, 1H), 1.05 (s, 9H), 0.98 (s, 3H), 0.89 (d, J = 6.6 Hz, 3H), 0.856 (d, J = 6.6 Hz, 3H), 0851 (d, J = 6.6 Hz, 3H), 0.64 (s, 3H); ${ }^{13} \mathrm{C}$ NMR (62.5 MHz, $\mathrm{CDCl}_{3}$ ) $\delta$ 141.3, 135.9 (4C), 134.9 (2C), 129.6 (2C), 127.6 (4C), 121.3, 73.4, 56.9, 56.3, 50.2, 42.7, 42.4, 39.9, 39.7, 37.4, 36.6, 36.4, 35.9, 32.0 (3C), 28.4, 28.1, 27.2 (3C), 24.4, 24.0, 23.0, 22.8, 21.2, 19.6, 19.3, 18.9, 12.0.

To a solution of compound 1a (14.5 g, $23.2 \mathrm{mmol})$ in dichloromethane (100 ml) was added, by small portions, an excess of $m$-CPBA $(85 \%, 5.7 \mathrm{~g}, 28 \mathrm{mmol})$ at room temperature. The reaction mixture was stirred overnight at room temperature. It was then washed with aqueous $\mathrm{Na}_{2} \mathrm{CO}_{3}$, water and brine. After drying over $\mathrm{Na}_{2} \mathrm{SO}_{4}$, the evaporation of the solvent under reduced pressure afforded $13.8 \mathrm{~g}$ (93\%) of the mixture of epoxides $\mathbf{1 b}$ (two diastereoisomers, $\alpha$ and $\beta$ ). A diastereoisomeric ratio $\alpha: \beta=2.7: 1$ was determined by ${ }^{1} \mathrm{H}$ NMR (300 $\mathrm{MHz}$, $\left.\mathrm{Cl}_{3} \mathrm{CD}\right)$ analysis of the crude product, integrating the $\mathrm{C} 3-\mathrm{H}$ signals at $\delta 3.94 \mathrm{ppm}(\mathrm{m}, 0.73 \mathrm{H}$, $\alpha$-epoxide) and $\delta 3.64 \mathrm{ppm}$ (m, 0.27H, $\beta$-epoxide). The $\mathrm{C} 6-\mathrm{H}$ (oxiranic proton) signals appeared, overlapping, at $\delta$ 2.69-2.77 ppm (m, 1H). To a solution of crude $\mathbf{1 b}$ (13.79 g, 21.5 $\mathrm{mmol})$ in acetone-dichloromethane $(6: 4,200 \mathrm{ml})$ stirred at $50^{\circ} \mathrm{C}$ was slowly added (addition over $3 \mathrm{~h}$ ) an excess of Jones reagent (freshly prepared from $5.1 \mathrm{~g}$ of $\mathrm{CrO}_{3}, 15 \mathrm{ml} \mathrm{of} \mathrm{H}_{2} \mathrm{O}$ and $4.3 \mathrm{ml}$ of concentrated sulfuric acid). The reaction mixture was then cooled to room temperature and 2-propanol was added until the reaction mixture turned blue. The reaction mixture was filtered, and evaporated under reduced pressure. The residue was dissolved in dichloromethane, washed with a saturated solution of oxalic acid and then with brine. After drying over $\mathrm{Na}_{2} \mathrm{SO}_{4}$, the solvent was evaporated under vacuum. The crude product was purified by chromatography on silica gel. The column was first eluted with petroleum ether and then with a 99:1 mixture of petroleum ether-diethyl ether. The fractions eluted with the mixture afforded $9.2 \mathrm{~g}$ of compound 2 as a white solid, mp $119-122^{\circ} \mathrm{C}$. Recristallization from $\mathrm{CH}_{2} \mathrm{Cl}_{2}-\mathrm{CH}_{3} \mathrm{CN}$ afforded $8.7 \mathrm{~g}$ (60\% yield) of 2 , white crystals mp. $122-123^{\circ} \mathrm{C} ;[\alpha]^{25} \mathrm{D}$ +48.16 (c 1, $\left.\mathrm{CHCl}_{3}\right), \mathrm{MS} \mathrm{ES}+695.4[\mathrm{M}+\mathrm{Na}]^{+} \mathrm{C}_{43} \mathrm{H}_{64} \mathrm{O}_{4} \mathrm{Si},{ }^{1} \mathrm{H}$ NMR (300 MHz, $\left.\mathrm{CDCl}_{3}\right)$ $\delta 7.63$ (m, 4H), 7.39 (m, 6H), 4.37 (br s 1H), 2.95 (dd, J = 13.2, J = 3.5 Hz, 1H), 2.34 (br d, J = 13.2 Hz, 1H), 2.25 (dd, J = 16.9, J = 3.2 Hz, 1H), 2.15 (dd, J = 16.9, J = 6.1 Hz, 1H), 1.08 (s, 3H), 1.03 (s, 9H), 0.88 (d, J = 6.6 Hz, 3H), 0.85 (br d, J = 6.6 Hz, 6H), 0.67 (s, 3H); ${ }^{13} \mathrm{C}$ NMR (62.9 MHz, $\mathrm{CDCl}_{3}$ ) 217.4, 178.3, 136.0 (2C), 135.8 (2C), 134.1, 133.8, 129.84, 129.76, 127.7 (4C), 72.5, 56.1, 55.2, 52.6, 46.8, 42.7, 42.0, 40.0, 39.6, 36.1, 35.9, 35.7, 34.6, 34.1, 28.6, 28.12, 28.05, 27.0, 24.5, 23.9, 23.2, 22.9, 22.7, 19.3, 18.7, 18.0, 11.8 . 
Thionyl chloride (3 ml, $41.1 \mathrm{mmol}$ ) was added to an anhydrous solution of the keto-acid 2 (6 g, $8.9 \mathrm{mmol}$ ) in ether-DMF (60 ml-6 ml). The reaction mixture was stirred for $1 \mathrm{~h}$ at room temperature and then poured onto ice and extracted with ether. The organic phase was washed with water and brine, dried $\left(\mathrm{Na}_{2} \mathrm{SO}_{4}\right)$ and evaporated to afford $6 \mathrm{~g}$ (97\% yield) of compound 2a; ${ }^{1} \mathrm{H}$ NMR (300 MHz, $\left.\mathrm{CDCl}_{3}\right) \delta 7.63(\mathrm{~m}, 4 \mathrm{H}), 7.39(\mathrm{~m}, 6 \mathrm{H}), 4.37$ (m, 1H), 3.01 (dd, J = 19.3, J = 5.2 Hz, 1H), 2.82 (dd, $\mathrm{J}=13.6, \mathrm{~J}=3.7 \mathrm{~Hz}, 1 \mathrm{H}$ ), 2.81 (dd, $\mathrm{J}=19.3, \mathrm{~J}=3.3 \mathrm{~Hz}, 1 \mathrm{H}$ ), 2.36 (br d, J = 13.6 Hz, 1H), 1.06 (s, 3H), 1.03 (s, 9H), 0.88 (d, J = 6.8 Hz, 3H), 0.855 (d, J = $6.6 \mathrm{~Hz}, 3 \mathrm{H}), 0.85$ (d, J = 6.6 Hz, 3H), 0.66 (s, 3H); ${ }^{13} \mathrm{C}$ NMR (62.5 MHz, $\left.\mathrm{CDCl}_{3}\right) \delta 217.0$, 173.8, 136.0 (2C), 135.8 (2C), 134.1, 133.6, 129.9, 129.8, 127.79 (2C), 127.74 (2C), 72.3, 56.2, 53.7, 52.4, 48.0, 46.6, 42.7, 41.3, 39.9, 39.6, 37.1, 36.1, 35.8, 33.8, 28.5, 28.1, 27.9, 27.0 (3C), 24.8, 23.9, 23.2, 22.9, 22.6, 19.3, 18.6, 18.0, 11.6.

A solution of sodium azide $(1.8 \mathrm{~g}, 29.5 \mathrm{mmol})$ in water $(18 \mathrm{ml})$ was added to a solution of the acid chloride $2 \mathrm{a}(6.0 \mathrm{~g}, 8.7 \mathrm{mmol})$ in acetone $(120 \mathrm{ml})$. The reaction mixture was stirred at room temperature for 30 minutes (water $(9 \mathrm{ml})$ was added after 15 minutes). The reaction mixture was then poured onto ice and extracted with ether. The organic layer was washed with water and brine, dried $\left(\mathrm{Na}_{2} \mathrm{SO}_{4}\right)$ and evaporated under reduced pressure to afford $5.58 \mathrm{~g}$ (93\% yield) of compound $\mathbf{2 b} ;{ }^{1} \mathrm{H}$ NMR (300 MHz, $\mathrm{CDCl}_{3}$ ) $\delta 7.63$ (m, 4H), 7.39 (m, 6H), 4.38 (m, 1H), 2.95 (dd, J = 13.4, J = 3.7 Hz, 1H), 1.06 (s, 3H), 1.03 (s, 9H), 0.88 (d, J = $6.8 \mathrm{~Hz}$, 3H), 0.853 (d, J = 6.6 Hz, 3H), 0.849 (3H, d, J = 6.6 Hz, 3H), 0.66 (s, 3H); ${ }^{13} \mathrm{C}$ NMR (62.9 $\left.\mathrm{MHz}, \mathrm{CDCl}_{3}\right) \delta 217.3,180.5,136.0$ (2C), 135.8, 134.1, 133.7, 129.86, 129.78, 127.7 (4C), 72.4, 56.1, 54.4, 52.5, 46.8, 42.7, 41.6, 39.9, 39.6, 37.0, 36.1, 35.9, 35.8, 34.0, 28.6, 28.1, 28.0, 27.0 (3C), 24.8, 23.8, 23.2, 22.9, 22.7, 19.3, 18.7, 18.0, 11.7.

A solution of the acyl-azide $2 \mathbf{b}(5.58 \mathrm{~g}, 8.0 \mathrm{mmol})$ in toluene $(25 \mathrm{ml})$ was stirred at $80^{\circ} \mathrm{C}$ for $30 \mathrm{~min}$. Then the solvent was evaporated under vacuum (at room temperature) affording $4.9 \mathrm{~g}$ (92\% yield) of compound 2c (white solid); $[\alpha]^{25}=+62.47$ (c 1, $\mathrm{CHCl}_{3}$ ); ${ }^{1} \mathrm{H} \mathrm{NMR}(250 \mathrm{MHz}$, $\left.\mathrm{CDCl}_{3}\right) \delta 7.64(\mathrm{~m}, 4 \mathrm{H}), 7.40(\mathrm{~m}, 6 \mathrm{H}), 4.42$ (m, 1H), 3.36 (dd, J = 13.9 Hz, J = 2.2 Hz, 1H), 3.21 (dd, $\mathrm{J}=13.9 \mathrm{~Hz}, \mathrm{~J}=1.9 \mathrm{~Hz}, 1 \mathrm{H}$ ), 3.06 (dd, J = 13.4 Hz, J = $3.5 \mathrm{~Hz}, 1 \mathrm{H}$ ), 2.43 (br d, J = $13.4 \mathrm{~Hz}, 1 \mathrm{H}$ ), 1.04 (s, 9H), 1.02 (s, 3H), 0.89 (d, J = 6.5 Hz, 3H), 0.86 (br d, J = 6.6 Hz, 6H), 0.66 (s, 3H); ${ }^{13} \mathrm{C}$ NMR (62.5 MHz, $\left.\mathrm{CDCl}_{3}\right) \delta 216.6,136.0,135.8,134.1,133.7,129.9,129.8$, 127.79, 127.75, 121.8, 72.4, 56.4, 52.3, 51.9, 46.7, 43.4, 42.4, 39.84, 39.81, 39.6, 39.0, 36.1, 35.9, 33.9 28.5, 28.1, 28.0, 27.0, 24.2, 23.9, 22.93, 22.86, 22.7, 19.4, 18.7, 17.9, 12.0.

Neutral alumina ( $3 \mathrm{~g}$ ) was added to a solution of the isocyanate $2 \mathrm{c}(4 \mathrm{~g}, 5.97 \mathrm{mmol})$ in petroleum ether $(40 \mathrm{ml})$. The reaction mixture was stirred at room temperature for $3 \mathrm{~h}$ and 
then filtered. The alumina was washed with petroleum ether. The solvent was evaporated under vacuum to afford $2.8 \mathrm{~g}$ (75\% yield) of compound 3; $[\alpha]^{25}{ }_{\mathrm{D}}-36.20$ (c 1, $\mathrm{CHCl}_{3}$ ); ${ }^{1} \mathrm{H}$ NMR (300 MHz, $\left.\mathrm{CDCl}_{3}\right) \delta 7.66(\mathrm{~m}, 4 \mathrm{H}), 7.36(\mathrm{~m}, 6 \mathrm{H}), 3.70(\mathrm{~m}, 1 \mathrm{H}), 3.61$ (ddd, J = 17.6, J = 5.2, $\mathrm{J}=2.1 \mathrm{~Hz}, 1 \mathrm{H}$ ), 2.85 (ddd, $\mathrm{J}=17.6, \mathrm{~J}=10.5, \mathrm{~J}=3.1 \mathrm{~Hz}, 1 \mathrm{H}$ ), 2.65 (br dd, $\mathrm{J}=13.5, \mathrm{~J}=$ 11.2, 1H), 2.53 (dd, J = 13.5, J = 5.1 Hz, 1H), 1.08 (s, 3H), 1.05 (s, 9H), 0.89 (d, J = $6.5 \mathrm{~Hz}$, 3H), $0.856(\mathrm{~d}, \mathrm{~J}=6.6 \mathrm{~Hz}, 3 \mathrm{H}), 0.852$ (d, J = 6.6 Hz, 3H), 0.65 (s, 3H); ${ }^{13} \mathrm{C}$ NMR (75 MHz, $\left.\mathrm{CDCl}_{3}\right) \delta 173.6,135.9$ (4C), 134.7, 134.2, 129.66, 129.63, 127.68 (2C), 127.61 (2C), 72.3, 55.9, 55.5, 54.4, 47.3, 45.8, 42.5, 39.61, 39.56, 38.4, 36.3, 35.8, 35.4, 31.3 (2C), 28.5, 28.1, 27.1 (3C), 23.92, 23.89, 22.9, 22.7, 20.3, 19.2, 19.1, 18.8, 12.1.

To a solution of 6-azacholesterol $3(1.54 \mathrm{~g}, 2.46 \mathrm{mmol})$ in petroleum ether $(75 \mathrm{ml})$ at $0^{\circ} \mathrm{C}$ was added (addition over $15 \mathrm{~min}$, under stirring) m-chloroperbenzoic acid (0.45 g, 84\%, 2.2 mmol) in methanol $(75 \mathrm{ml})$. The reaction mixture was stirred for $5 \mathrm{~min}$ at $0^{\circ} \mathrm{C}$ and then the solvent was evaporated. The residue was dissolved in dichloromethane and the solution was washed with aqueous sodium bicarbonate, then with water and dried over sodium sulfate. The solvent was evaporated. Chromatography of the crude product on silica gel eluting with petroleum ether-diethyl ether (98:2) afforded $1.2 \mathrm{~g}$ (76\% yield) of compound 4 ; $[\alpha]^{25}-44.39$ (c 1, $\left.\mathrm{CHCl}_{3}\right) ;{ }^{1} \mathrm{H}$ NMR (300 MHz, $\left.\mathrm{CDCl}_{3}\right) \delta 7.63(\mathrm{~m}, 4 \mathrm{H}), 7.37$ (m, 6H), $3.82(1 \mathrm{H}, \mathrm{m}, \mathrm{H}-3)$, 3.40 (dd, $\mathrm{J}=17.0, \mathrm{~J}=7.4 \mathrm{~Hz}, 1 \mathrm{H}$ ), 2.88 (dd, $\mathrm{J}=17.0$, $\mathrm{J}=9.9 \mathrm{~Hz}, 1 \mathrm{H}$ ), 2.32 (dd, $\mathrm{J}=12.4 \mathrm{~Hz}, \mathrm{~J}$ = $11.3 \mathrm{~Hz}, 1 \mathrm{H}), 1.94(\mathrm{br} \mathrm{d}, \mathrm{J}=12.4,1 \mathrm{H}), 1.14$ (s, 3H), 1.03 (s, 9H), 0.86 (d, J = 6.6 Hz, 3H), 0.851 (d, J = 6.6 Hz, 3H), 0.846 (d, J = 6.6 Hz, 3H), 0.59 (s, 3H); ${ }^{13} \mathrm{C}$ NMR (75 MHz, $\mathrm{CDCl}_{3}$ ) $\delta 135.84$ (2C), 135.80 (2C), 134.6, 134.2, 129.69, 129.66, 127.66 (2C), 127.62 (2C), 87.7, 70.2, 55.6, 55.1, 54.0, 42.7, 41.3, 40.9, 39.6, 39.4, 36.3, 35.8, 34.9, 32.5, 31.1, 30.5, 28.3, 28.1, 27.1 (3C), 23.9, 23.5, 22.9, 22.7, 20.2, 19.2, 18.7, 15.6, 12.1; HRMS (ES+) calcd. for $\mathrm{C}_{42} \mathrm{H}_{63} \mathrm{NO}_{2} \mathrm{Si}(\mathrm{M}+\mathrm{H})^{+}$642.4706, found 642.4708; Anal. Calcd. for $\mathrm{C}_{42} \mathrm{H}_{66} \mathrm{NO}_{2} \mathrm{SiBF}_{4}$ : C 78.75\%; H 9.89\%; N 2.18\%. Found: C 78.44\%; H 9.75\%; N 2.08\%.

To a solution of oxaziridine 4 (1.18 g, $1.84 \mathrm{mmol})$ in dichloromethane (8 ml) was added one equivalent of the Meerwein salt trimethyloxonium tetrafluoroborate (174 mg). The reaction mixture was stirred at room temperature for $2 \mathrm{~h}$ and then filtered. The solvent was evaporated under reduced pressure to afford $1.23 \mathrm{~g}$ (90 \% yield) of compound 5 as a white solid, mp 152$154^{\circ} \mathrm{C} ;[\alpha]^{25}-27.26$ (c 1, $\mathrm{CHCl}_{3}$ ); ${ }^{1} \mathrm{H}$ NMR (300 MHz, $\left.\mathrm{CDCl}_{3}\right) \delta 7.64$ (m, 4H), 7.41 (m, 6H), 4.19 (dd, $\mathrm{J}=14.6, \mathrm{~J}=7.7 \mathrm{~Hz}, 1 \mathrm{H}$ ), 3.71 (m, 1H), 3.31 (dd, $\mathrm{J}=14.6 \mathrm{~Hz}, \mathrm{~J}=10.8 \mathrm{~Hz}$, 1H), 3.18 (s, 3H), 2.60 (dd, $\mathrm{J}=12.8 \mathrm{~J}=11.4 \mathrm{~Hz}, 1 \mathrm{H}), 1.41$ (s, 3H), 1.07 (s, 9H), 0.86 (d, J = $6.1 \mathrm{~Hz}, 3 \mathrm{H}), 0.849$ (d, J = 6.6 Hz, 3H), 0.844 (d, J = 6.6 Hz, 3H), 0.63 (s, 3H); ${ }^{13} \mathrm{C}$ NMR $(75$ 
$\left.\mathrm{MHz}, \mathrm{CDCl}_{3}\right) \delta 135.9$ (2C), 135.7 (2C), 133.5, 133.2, 130.4, 130.2, 128.1 (2C), 127.9 (2C), 101.9, 70.3, 63.4, 55.4, 53.9, 48.3, 42.6, 39.6, 39.5, 38.8, 38.2, 36.2, 35.6, 33.8, 32.7, 29.9, 29.0, 28.1, 28.0, 26.9, 23.8, 22.87, 22.83, 22.6, 19.8, 19.0, 18.7, 15.0, 11.9; MS (ES+) m/z (\%) $657.2(1 \%)\left[\left(\mathrm{M}-\mathrm{F}_{4} \mathrm{~B}\right)^{+}\right], 414.8(100)$; Anal. calcd. for $\mathrm{C}_{43} \mathrm{H}_{66} \mathrm{NO}_{2} \mathrm{SiBF}_{4}\left(1 / 2 \mathrm{H}_{2} \mathrm{O}\right)$ : C 68.60\%; H 8.97\%; N 1.86\%. Found: C 68.63\%; H 8.81\%; N 1.82\%.

General procedure for asymmetric sulfoxidation (Table 1 and Scheme 2).

To a solution of the sulfide, $0.05 \mathrm{~N}$ in dichloromethane was rapidly added, under stirring, an equimolecular amount of the oxaziridinium 5 , as a $0.05 \mathrm{~N}$ solution in the same solvent at the appropriate temperature. The reactions carried out at room temperature were stirred for a few minutes (while a TLC checking and a potassium iodide test were performed) before workup. The reactions started at $-70^{\circ} \mathrm{C}$ (dry ice/ethanol bath) were stirred under argon while allowed to reach slowly room temperature (about $3 \mathrm{~h}$ ) before work up. In both cases, after removal of the solvent under reduced pressure, the sulfoxides were isolated by column chromatography (on silica gel or on alumina). The enantiomeric excesses were determined by HPLC using a CHIRALCEL OD column. They were validated with the racemic sulfoxides, prepared by standard oxidation ( $m$-CPBA) of the corresponding sulfides. HPLC conditions and the retention times for the enantiomers are given in pages S14-S33 


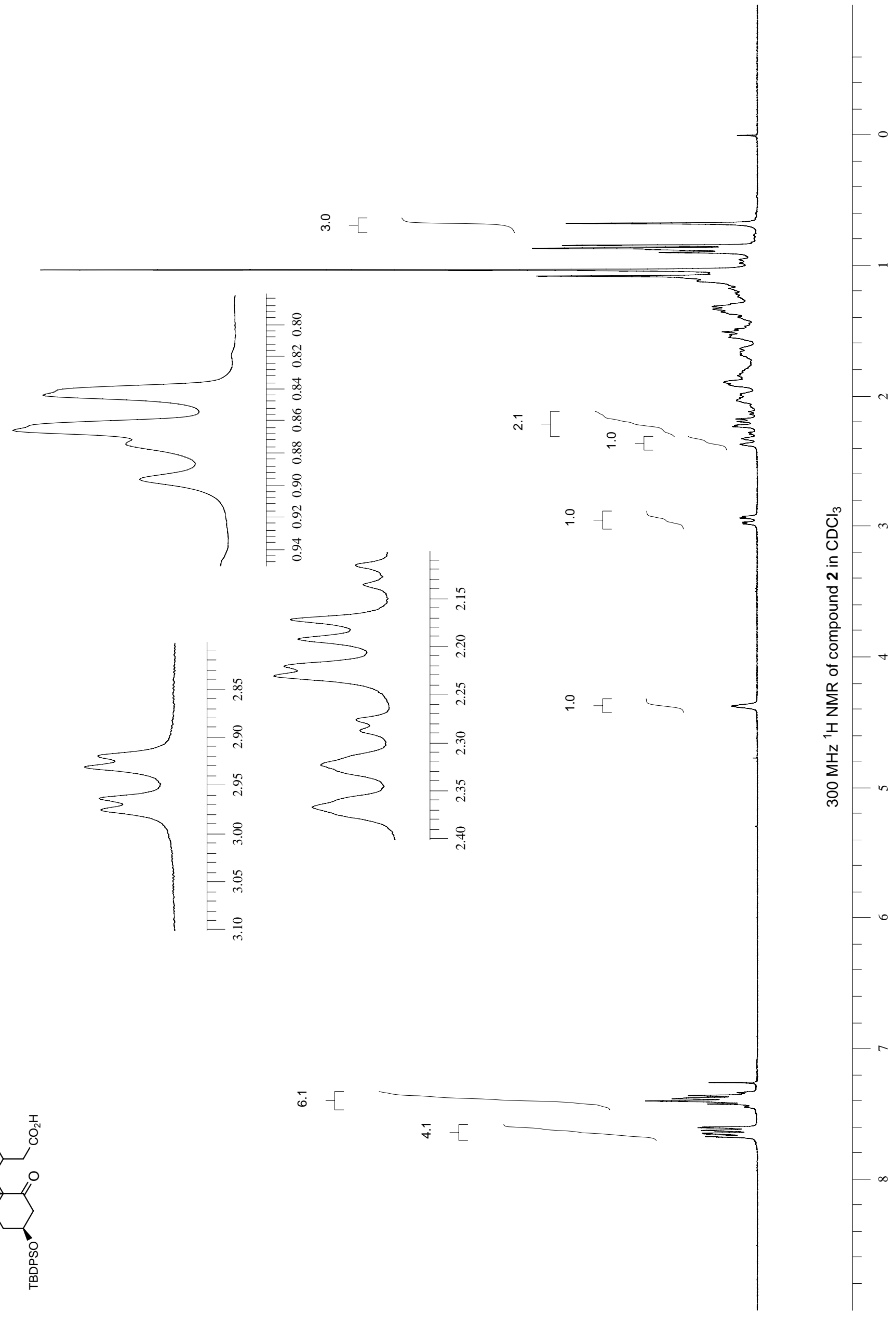



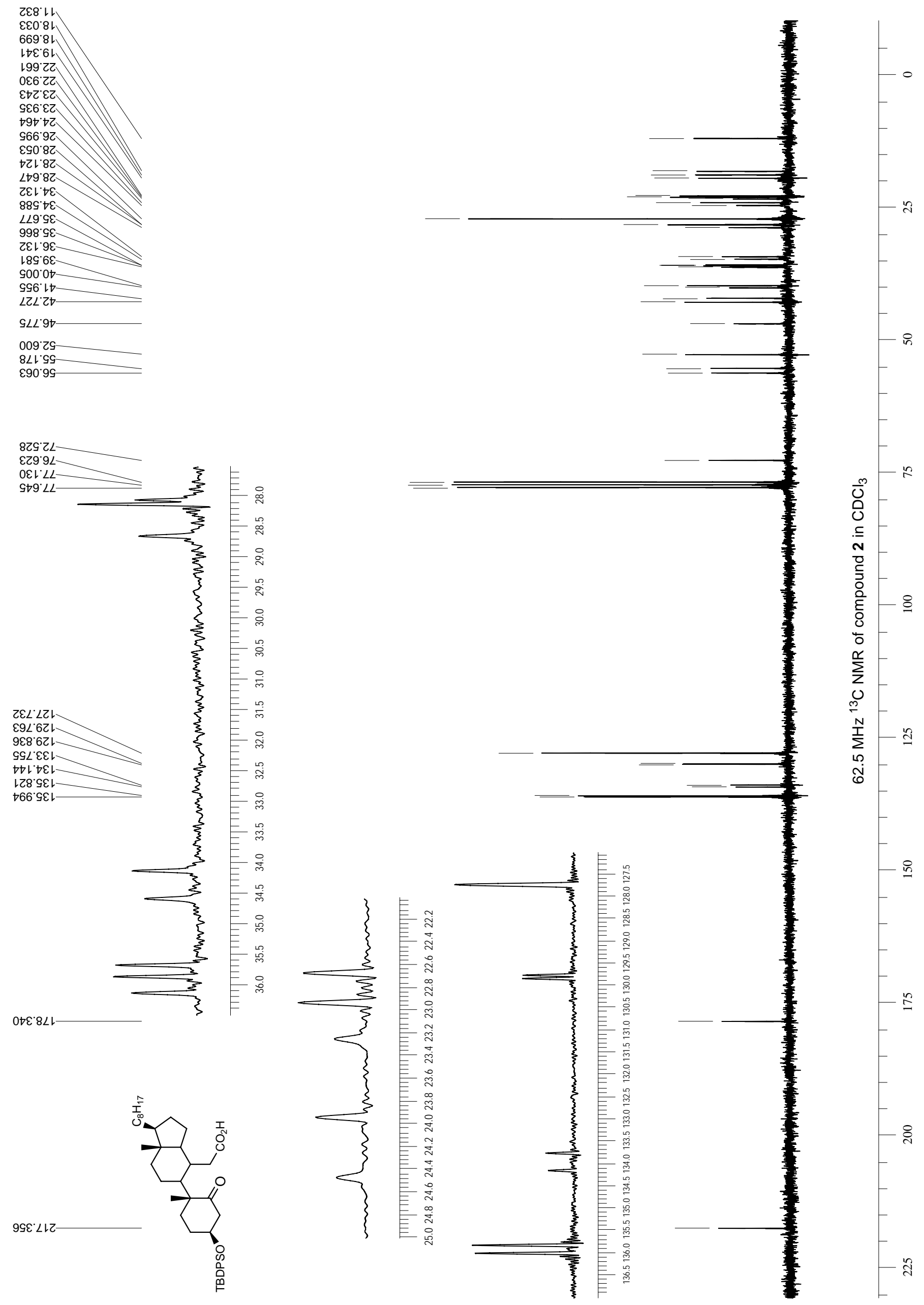

$99 \varepsilon^{\circ} \angle \tau Z$

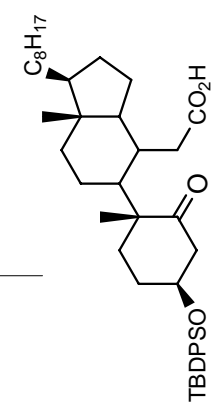



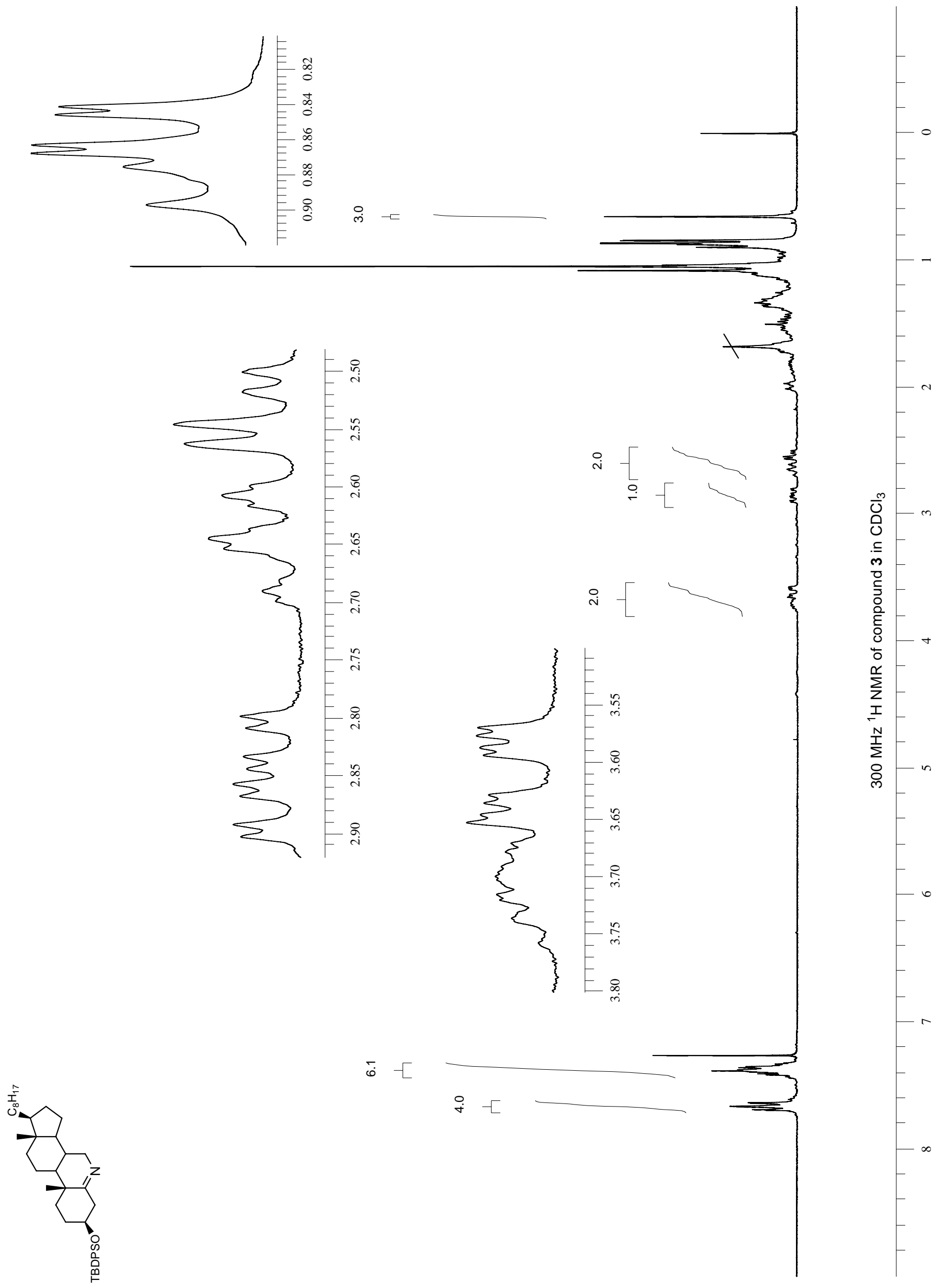
9S0' $2 T$

ยZ8. $8 T$

โ80 $6 \tau$

$6 \nabla 26 \mathrm{~T}$

ZLZ०Z

Z99'ZZ

งZ6'ZZ

ย68 $\varepsilon 乙$

โZ6 ${ }^{\circ} \varepsilon 乙$

$880^{\circ} \angle 2$

ย乙Т' $8 乙$

$\varepsilon 87.82$

$80 \varepsilon^{\prime} \tau \varepsilon$

$0 \angle \varepsilon^{\prime} \subseteq \varepsilon$

8Z8. $9 \varepsilon$

$96 Z^{\circ} 9 \varepsilon$

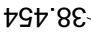

$6 \varsigma 9^{\circ} 6 \varepsilon$

ZT9.6E

OZS'Z๐

SGL'St

$90 \varepsilon \angle t$

$\nabla \angle \varepsilon^{\circ} \nabla G$

โ87' $9 S$

668 '

Z6Z'ZL

OIL'9L

SET' $L L$

ESG' LL

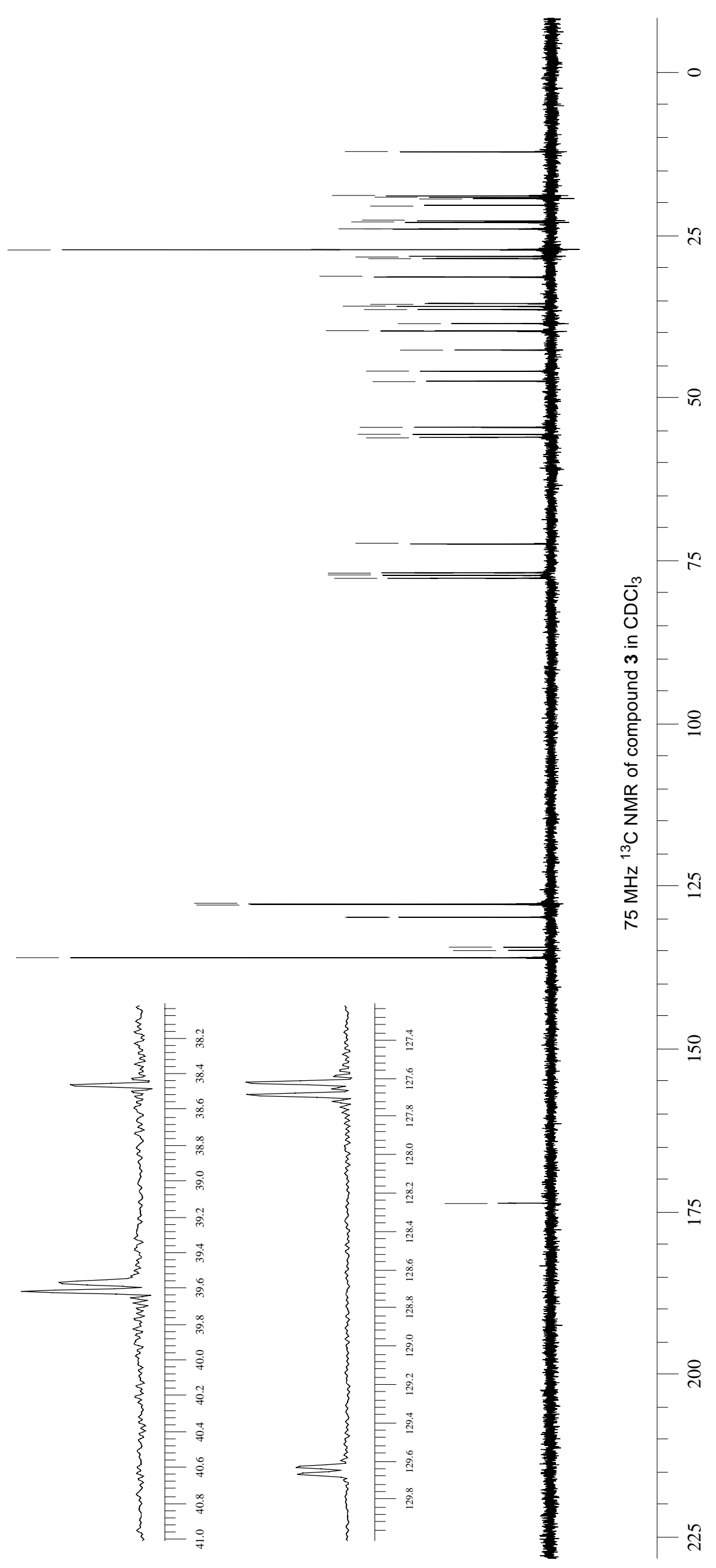

ET9 LZT

8L9'LZT

$\angle Z 9^{\circ} 6 Z \mathrm{~T}$

†99.6ZT

OSZ $\downarrow \varepsilon \tau$

$\tau \sqcup L ' \nabla \varepsilon \tau$

乙L8 SET

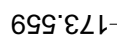
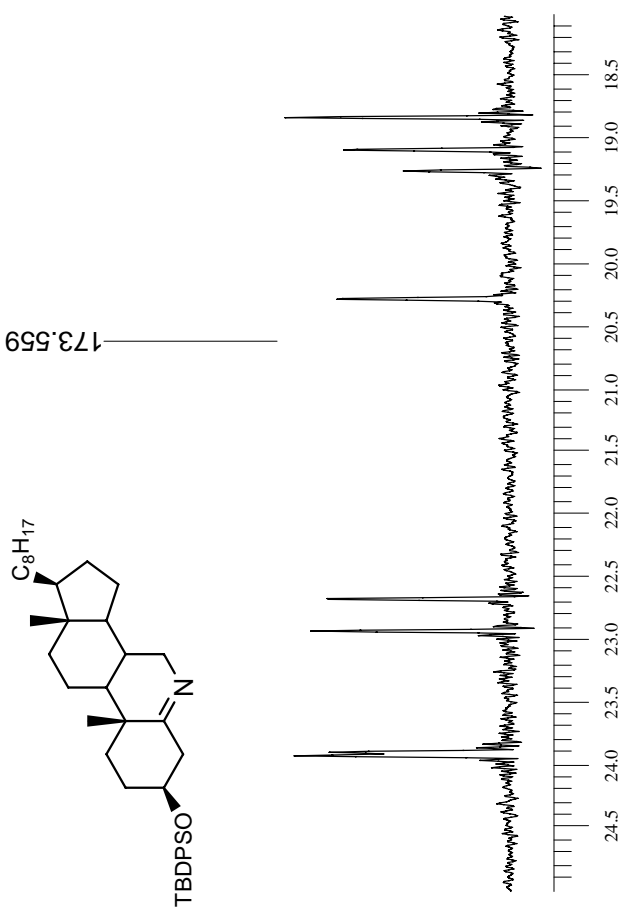


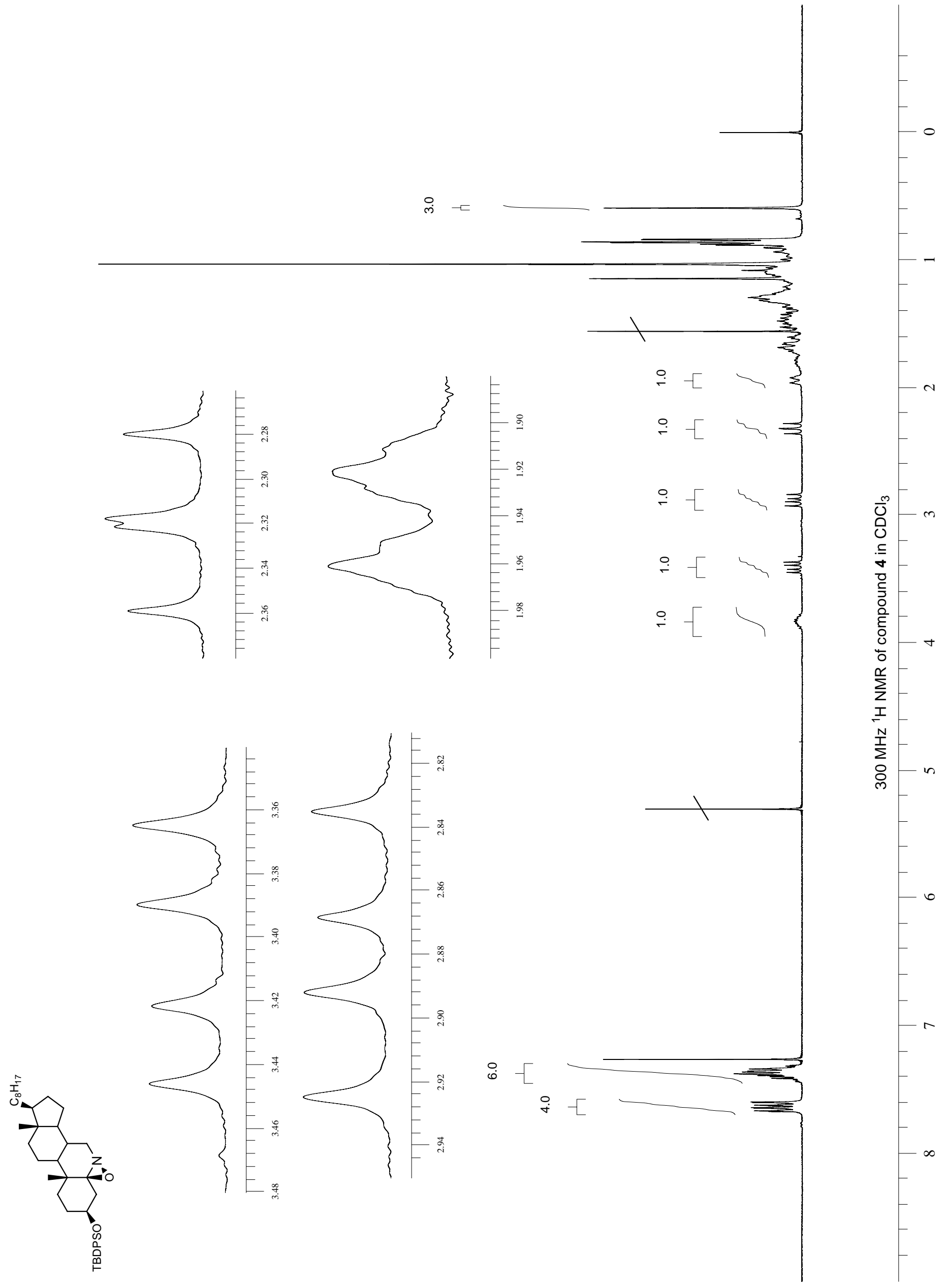


80T' $2 T$

$\angle 89 ' 9 T$

$0 S L \cdot 8 T$
$8 \gg Z \cdot 6 T$

$\varepsilon \angle T \cdot 0 z$

s99'zZ

$\angle T 6$ 'ZZ

$99 t^{\prime} \varepsilon z$

$926 . \varepsilon 2$

$\tau 60^{\circ} \angle 2$

LOT' 82

$6 \varepsilon \varepsilon \cdot 82$

$\mathrm{ZtG} \cdot 0 \varepsilon$

Z80' $โ \varepsilon$

โ6t' $2 \varepsilon$

$888^{\circ} \forall \varepsilon$

Z8L' $\mathrm{A \varepsilon}$

乙SZ' $9 \varepsilon$

ง8ะ. $6 \varepsilon$

S69' $6 \varepsilon$

$6880 \mathrm{t}$

$26 Z^{\prime} T t$

90L' $Z T$

000 't

\&90' 99

0t9 9

†G '0L-

60L' $9 L$

$9 \varepsilon{ }^{\prime} L L$

ZSG' $L L$

\&69 28

ZZ9. LZT

$999^{\circ} \angle Z L$
$999^{\circ} 6 Z \mathrm{~L}$

$\checkmark 69^{\circ} 62 \mathrm{~L}$

$9 Z{ }^{\circ} \nabla \varepsilon \tau$

५Z9" $\triangleright \varepsilon \tau$

ह08 ตET

LE8'งEโ-
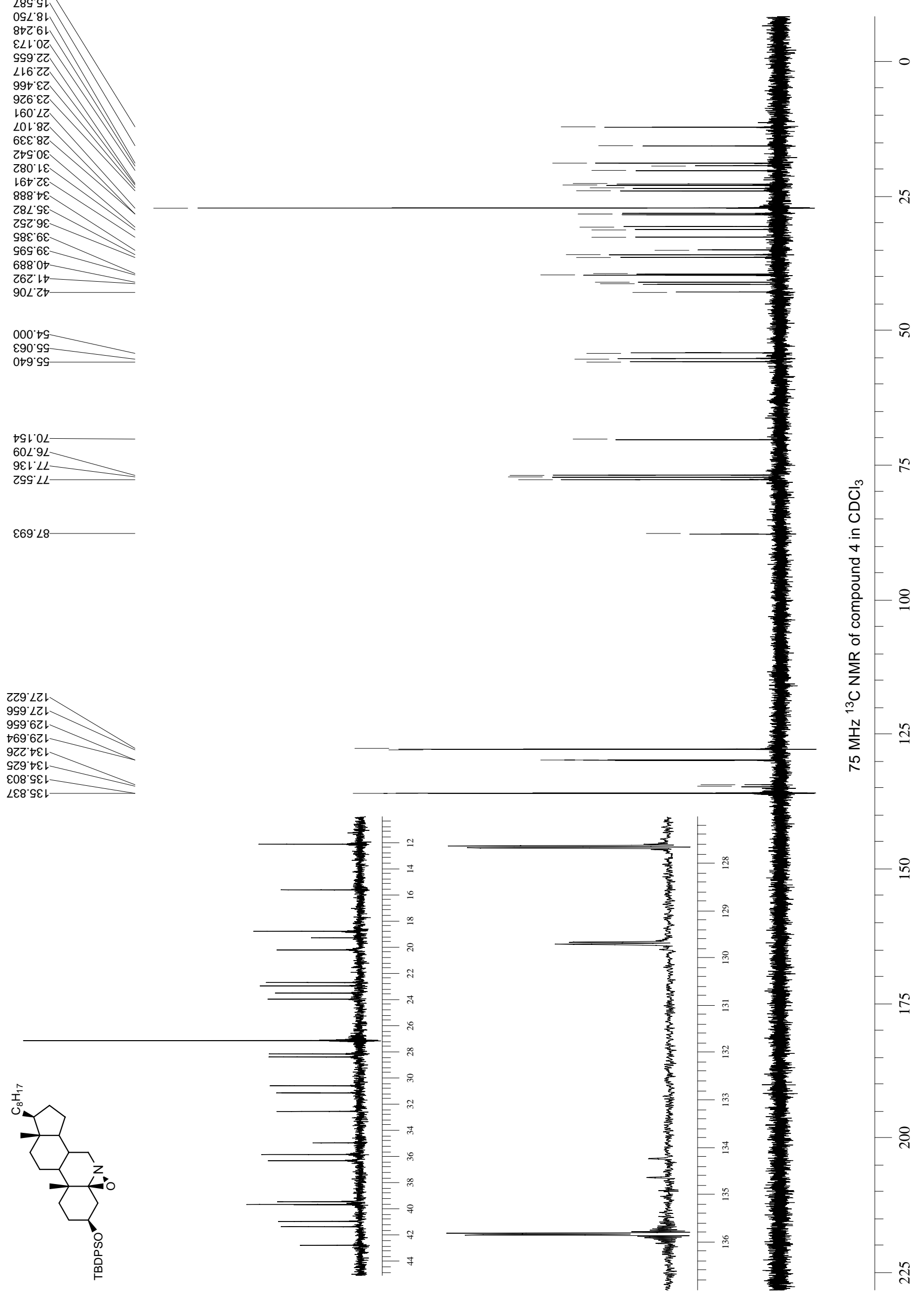


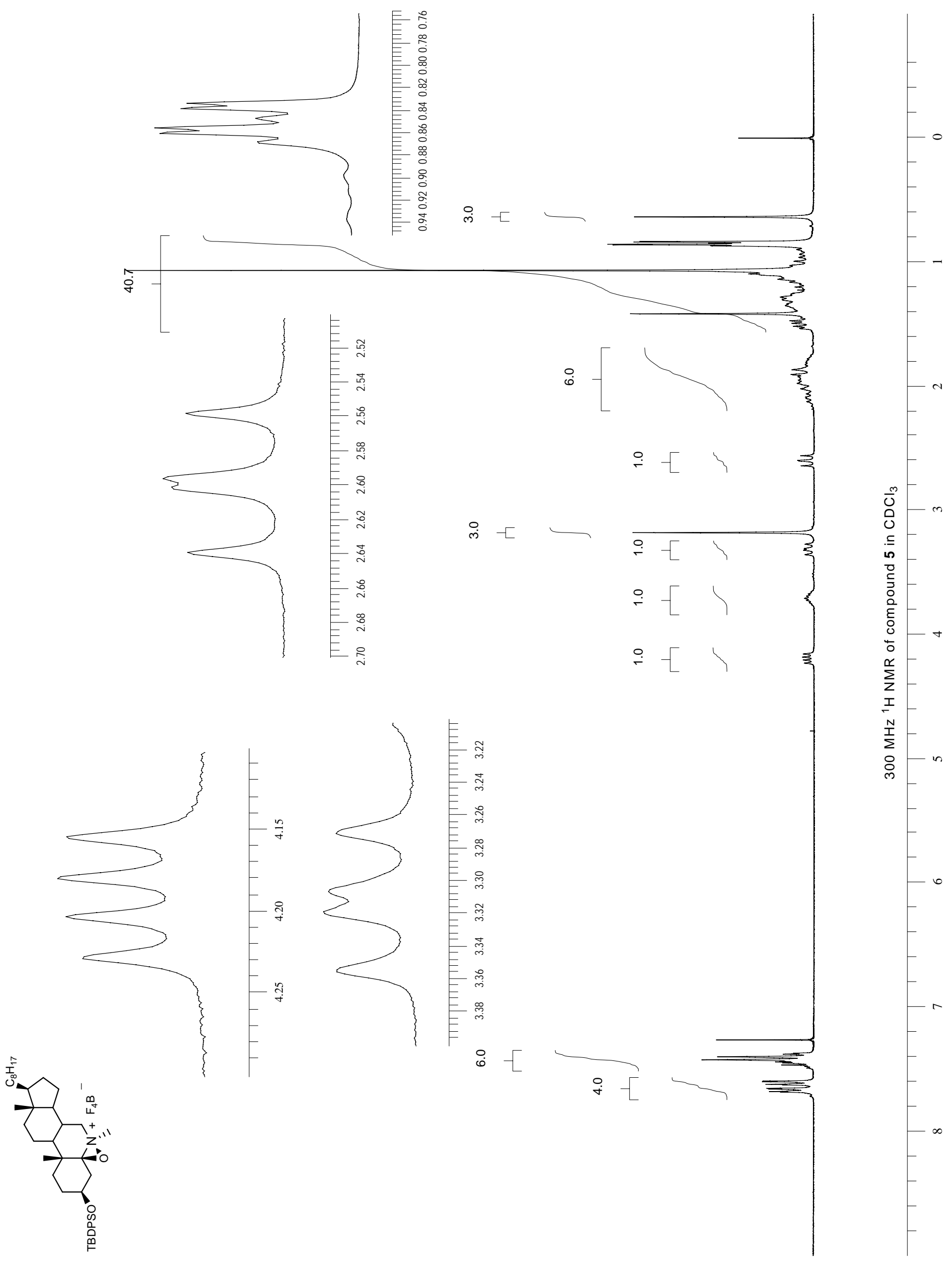




$$
1
$$




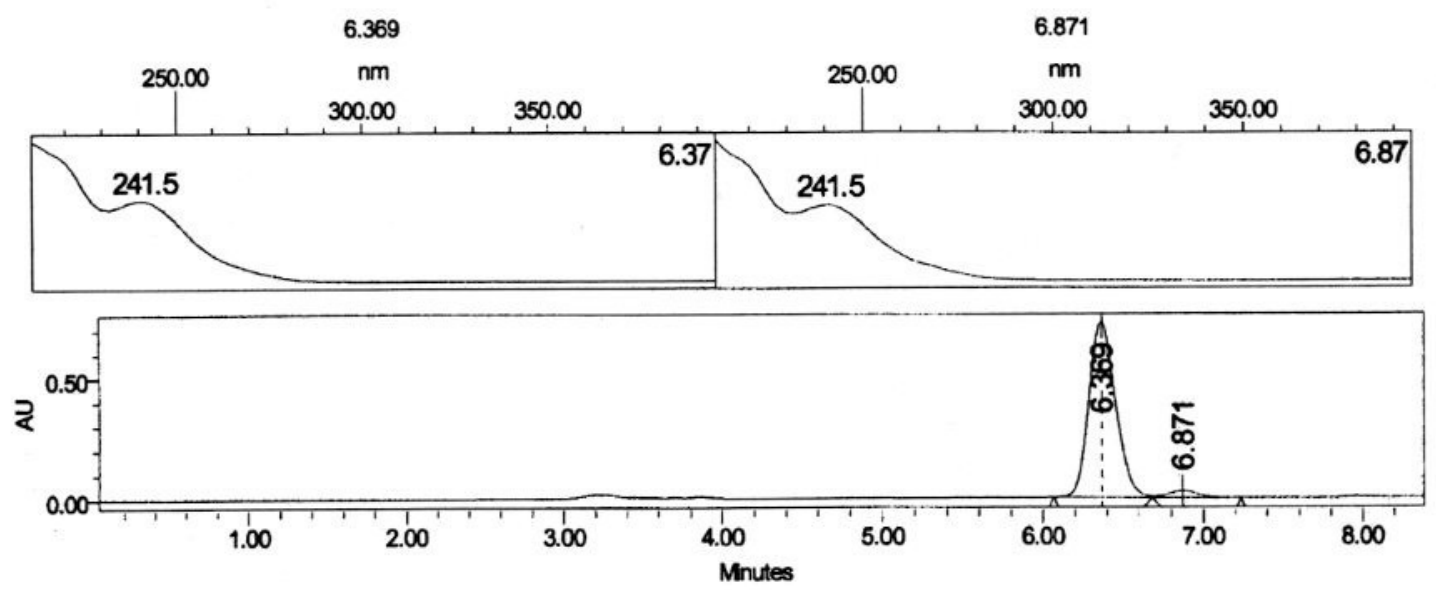

Colonne: $O D$

Hexane/lsopropand : $90 / 10$

Flow. $1 \mathrm{~m} / \mathrm{min}$

Proc. Orvl. Descr.: PDA $230.0 \mathrm{~mm}$

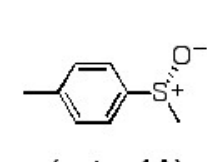

(entry 1A)

\begin{tabular}{|c|c|c|c|c|}
\hline \multicolumn{5}{|c|}{ Peak Results } \\
\hline & Rा & Area & Height & $\%$ Area \\
\hline 1 & 6.369 & 8317644 & 724184 & 95.93 \\
\hline 2 & 6.871 & 352662 & 28037 & 4.07 \\
\hline
\end{tabular}
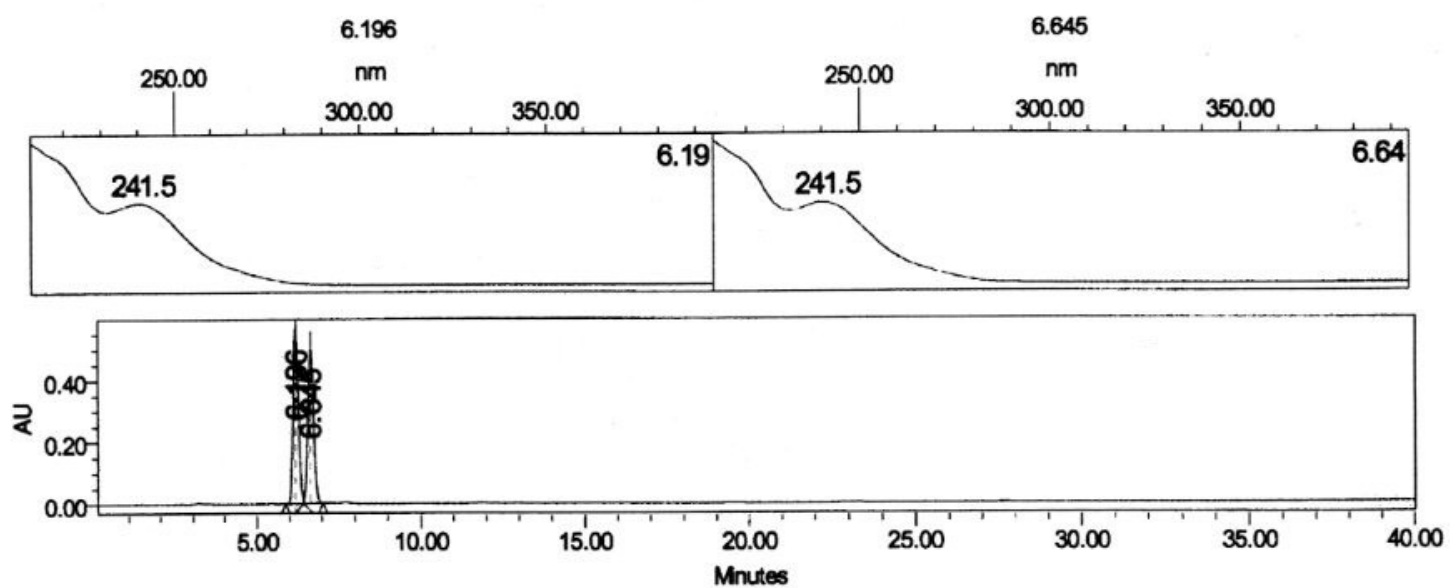

Colome: $O D$

Hexene/lsopropand : 90/10

Flow. $1 \mathrm{ml} / \mathrm{min}$

Proc. Orrl. Descr.: PDA $230.0 \mathrm{~mm}$

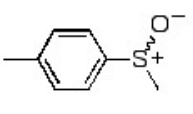

Peak Results

\begin{tabular}{|c|c|c|c|r|}
\hline & RT & Area & Height & \% Area \\
\hline 1 & 6.196 & 6289938 & 571561 & 50.35 \\
\hline 2 & 6.645 & 6201978 & 508298 & 49.65 \\
\hline
\end{tabular}



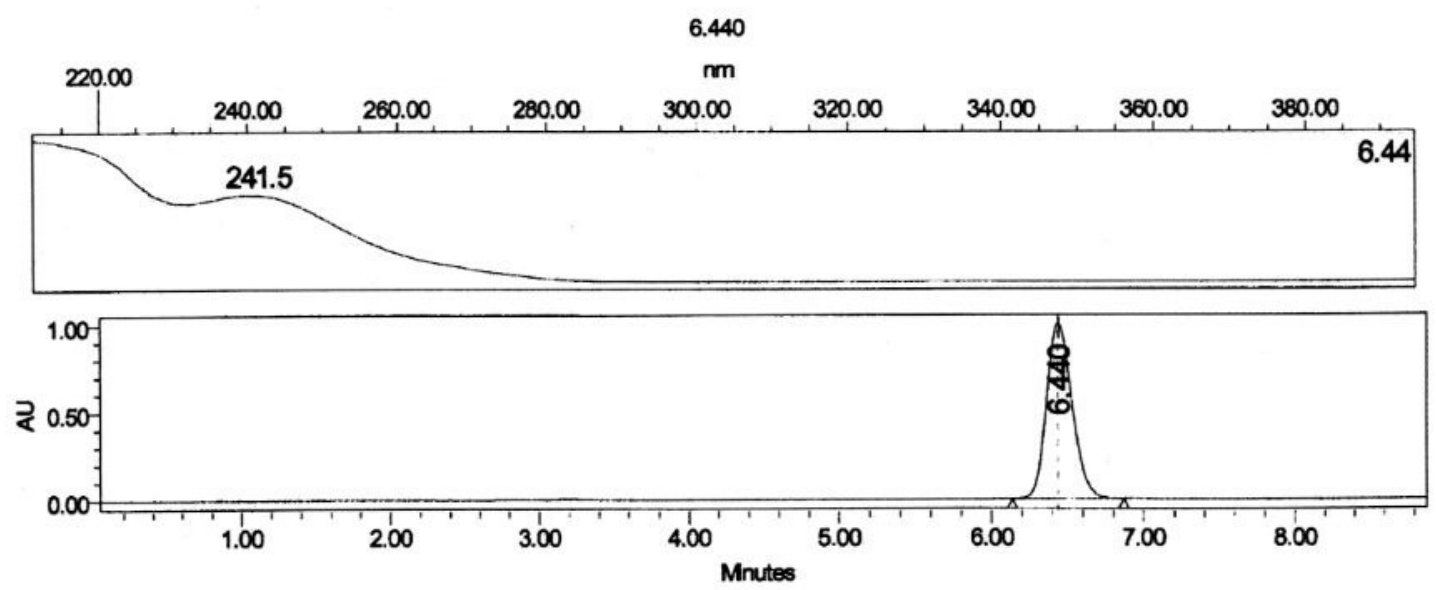

Colorne: OD

Hexane/sopropand : 90/10

Flow. $1 \mathrm{~m} / \mathrm{min}$

Proc. Arr. Descr.: PDA $230.0 \mathrm{~mm}$

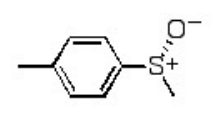

(entry 1B)

Peak Results

\begin{tabular}{|c|c|c|c|c|}
\hline \multicolumn{5}{|c|}{ Peak Results } \\
\hline & Rा & Area & Height & $\%$ Area \\
\hline 1 & 6.440 & 11674265 & 1004336 & 100.00 \\
\hline
\end{tabular}
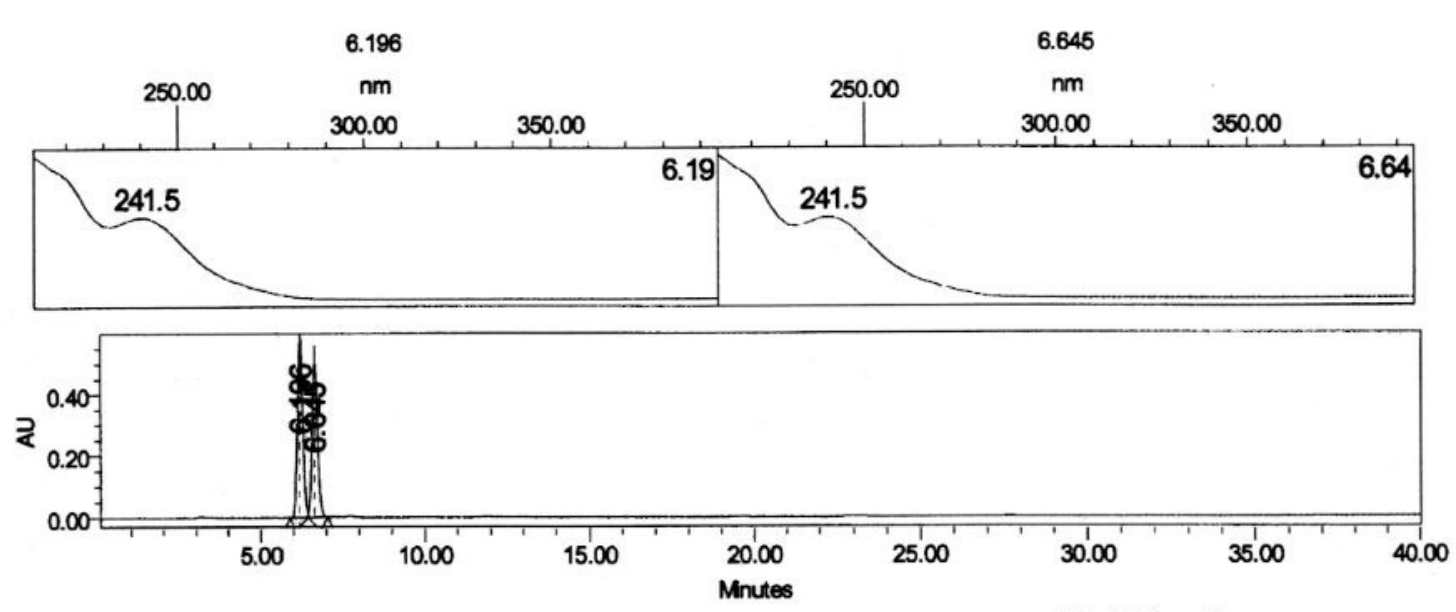

Colonne: OD

Hexane/sopropend : 90/10

Flow. $1 \mathrm{~m} / \mathrm{min}$

Proc. Ont. Descr.: PDA $230.0 \mathrm{~mm}$

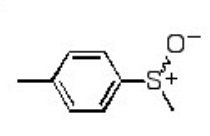

Peak Results

\begin{tabular}{|r|c|c|c|r|}
\hline & RT & Area & Height & $\%$ Area \\
\hline 1 & 6.196 & 6289938 & 571561 & 50.35 \\
\hline 2 & 6.645 & 6201978 & 508298 & 49.65 \\
\hline
\end{tabular}



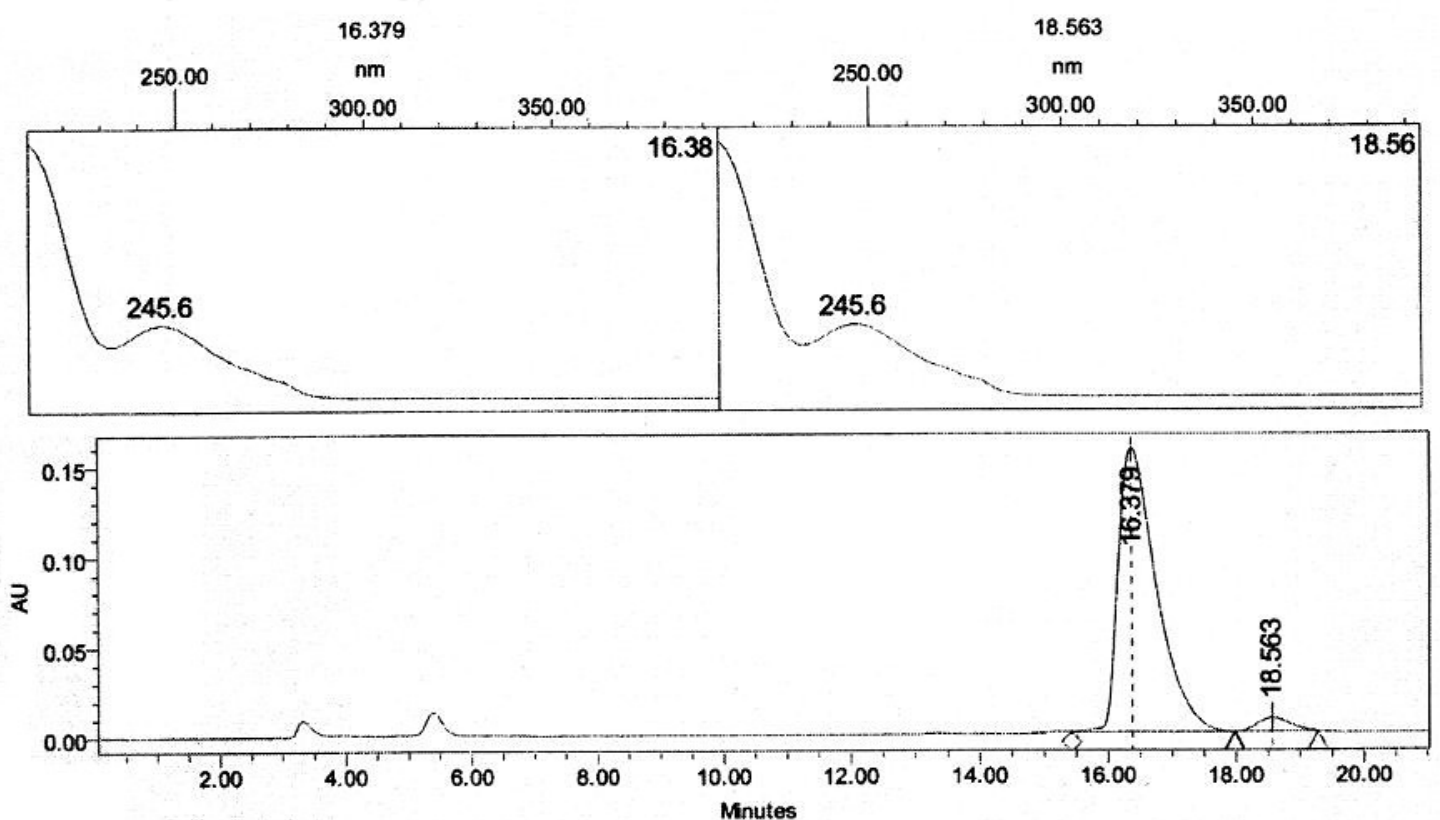

Processed Channel Descr. PDA $230.0 \mathrm{~nm}$

Colonne chirale: $\mathrm{OD}$

Hexane/lsopropanol: 95:05

How: $1 \mathrm{~m} / \mathrm{min}$
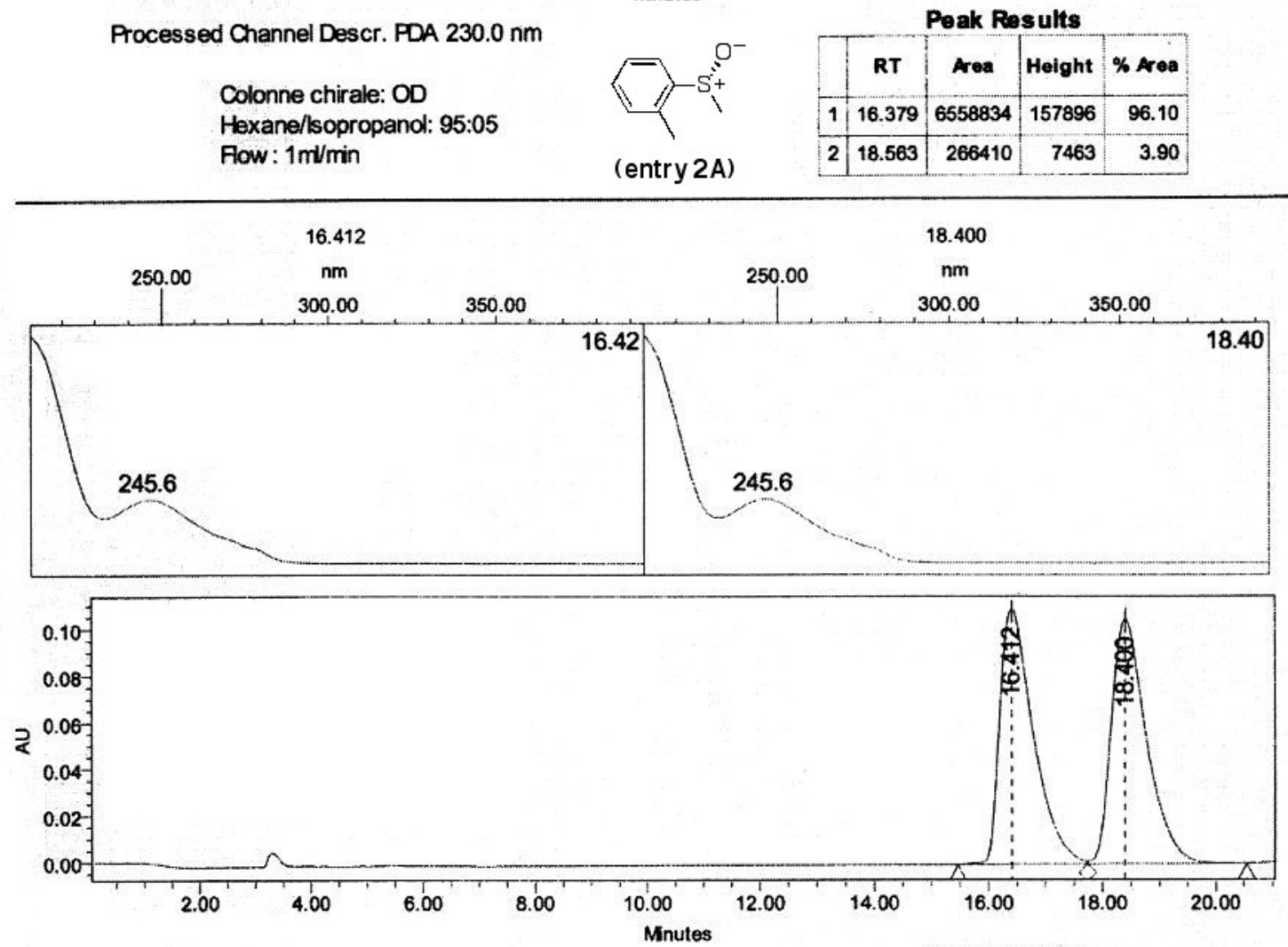

Processed Channel Descr. PDA $230.0 \mathrm{~nm}$

Colonne chirale: $\mathrm{OD}$

Hexane/lsopropanol: 95:05

Flow: $1 \mathrm{~m} / / \mathrm{min}$

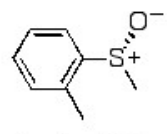

(entry 2A)

Peak Results

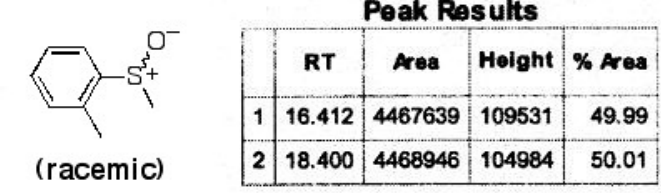



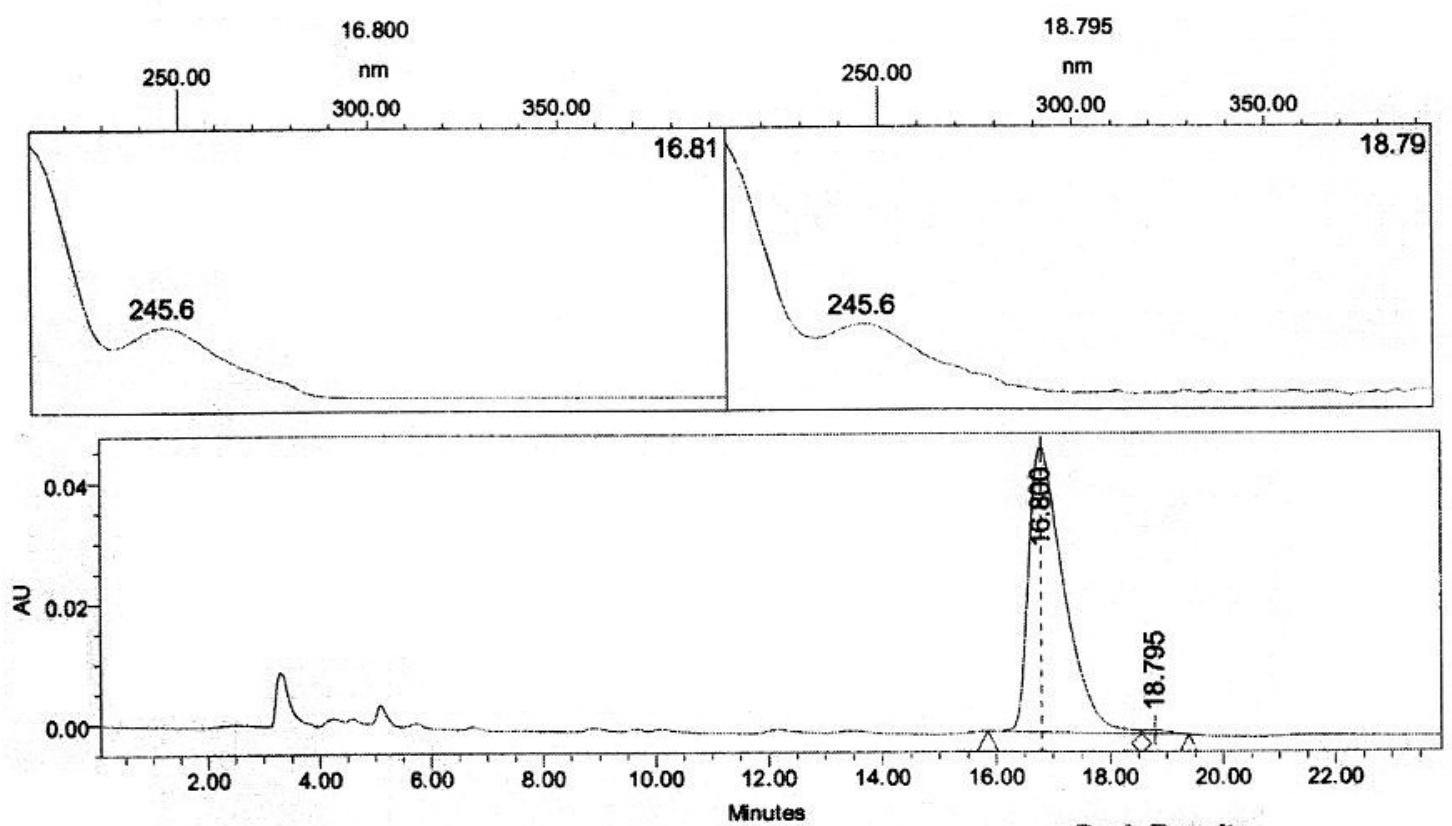

Processed Channel Descr. PDA $230.0 \mathrm{~nm}$

Colonne chirale: $O D$

Hexane/sopropanol: 95: 05

Fow: $1 \mathrm{~m} / \mathrm{min}$

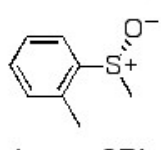

\begin{tabular}{|r|r|r|r|r|}
\hline & RT & Area & Helght & \% Area \\
\hline 1 & 16.800 & 2034065 & 47051 & 99.05 \\
\hline 2 & 18.795 & 19516 & 618 & 0.95 \\
\hline
\end{tabular}
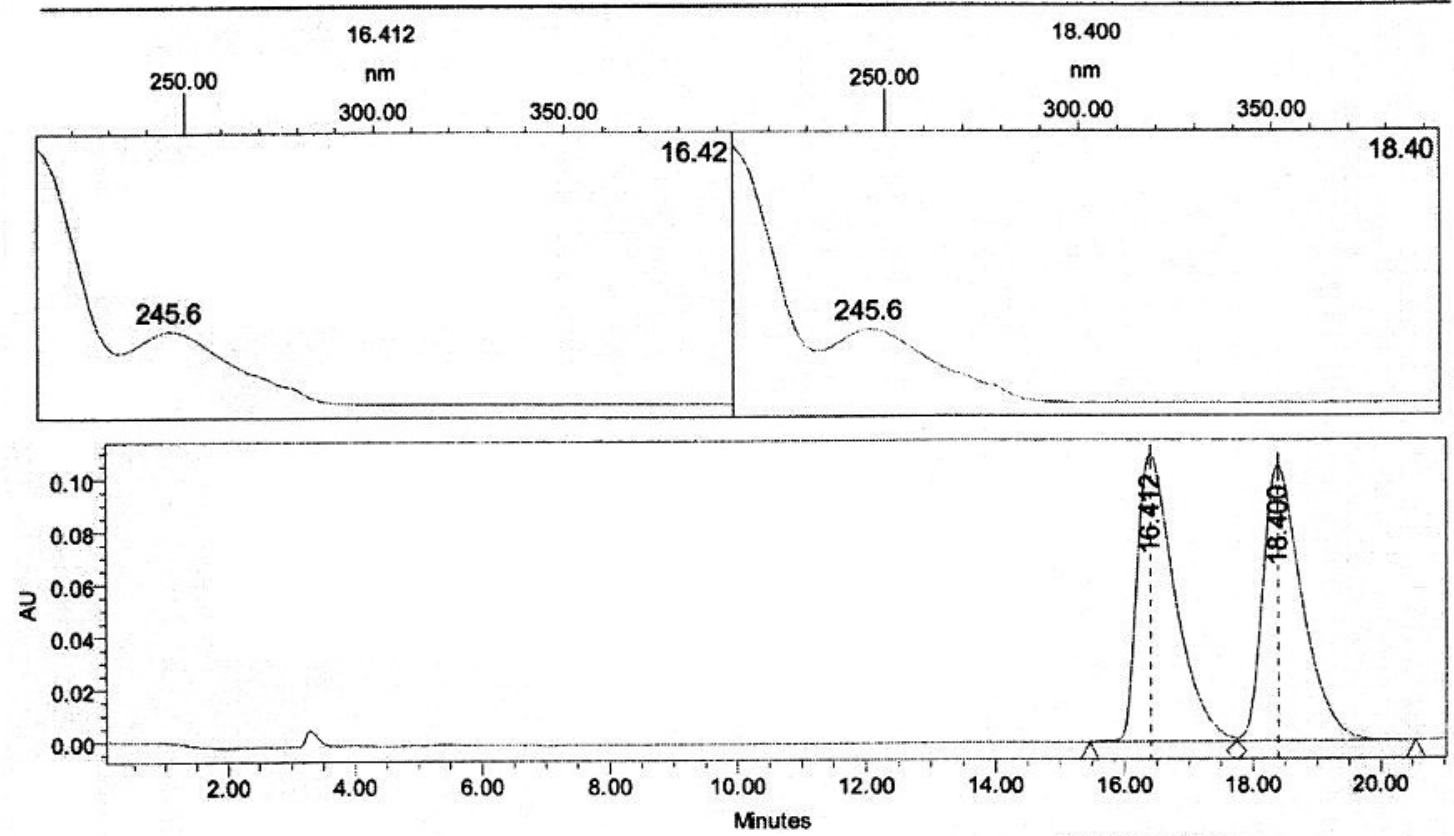

Processed Channel Descr. PDA $230.0 \mathrm{~nm}$

Colonne chirale: $O D$

Hexane/sopropanol: 95:05 Flow: $1 \mathrm{~m} / \mathrm{min}$

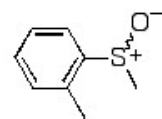

(racemic)
Peak Results

\begin{tabular}{|c|c|c|c|c|}
\hline & RT & Area & Height & \% Area \\
\hline 1 & 16.412 & 4467639 & 109531 & 49.99 \\
\hline 2 & 18.400 & 4468946 & 104984 & 50.01 \\
\hline
\end{tabular}



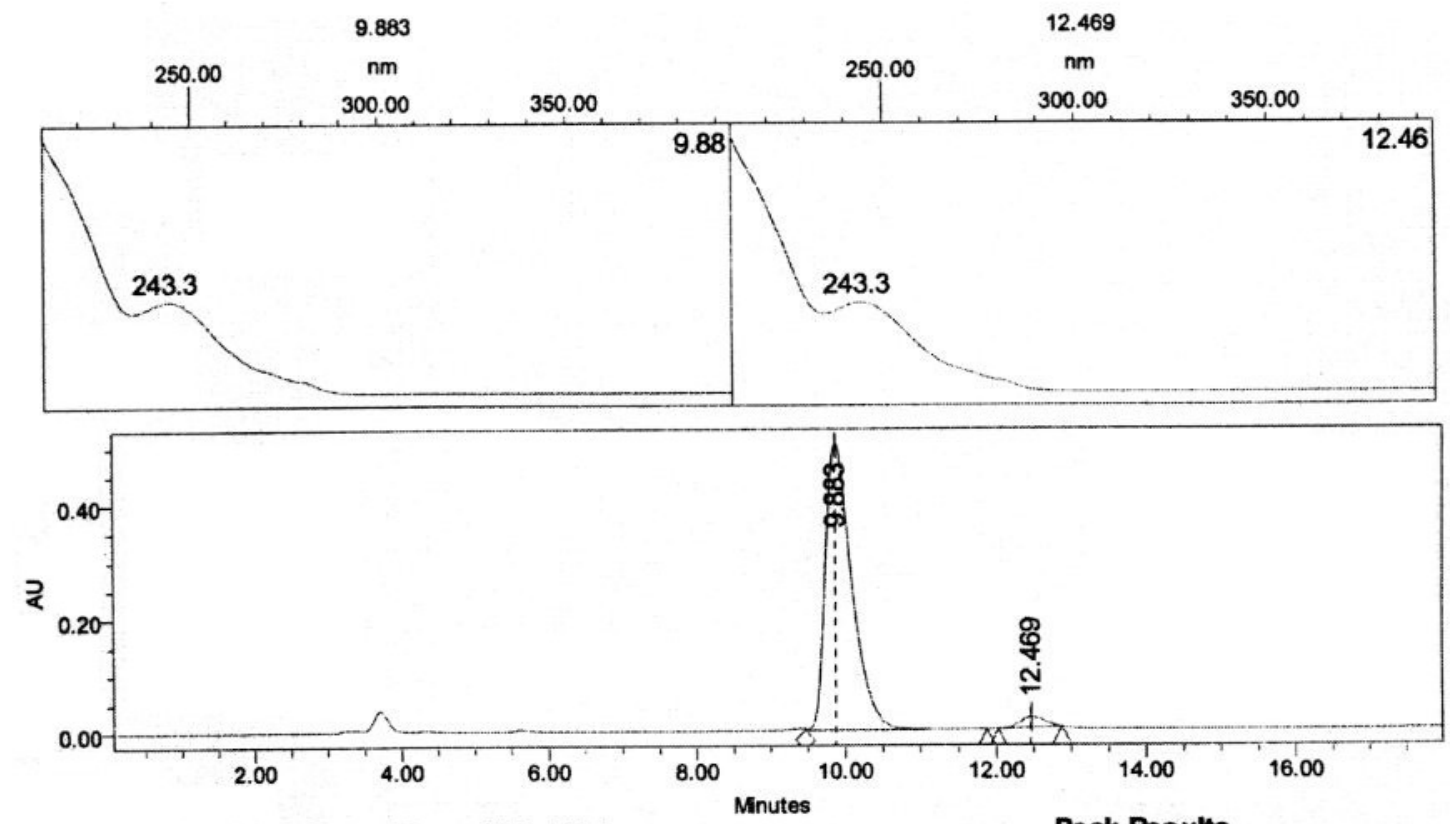

Processed Channel Descr. FDA $243.3 \mathrm{~nm}$

Colonne chirale: $O D$

Hexane/sopropanol: 90:10

Fow: $1 \mathrm{~m} / \mathrm{min}$

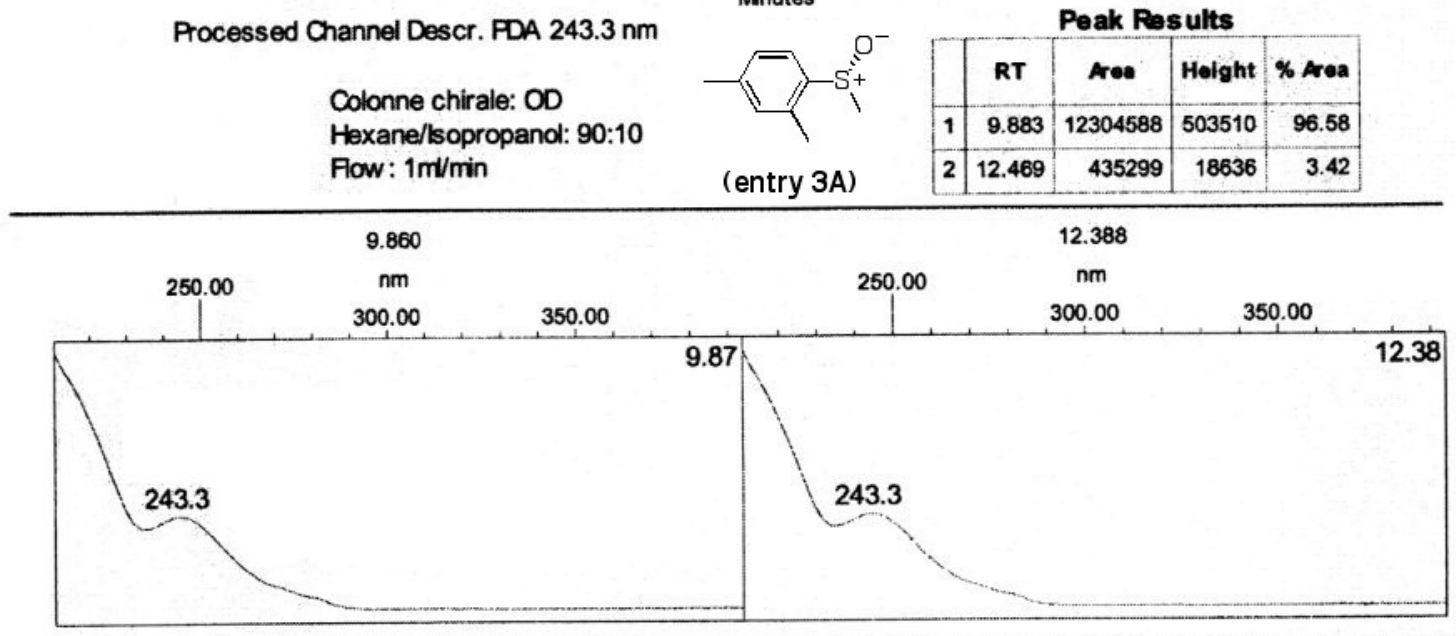

Peak Results

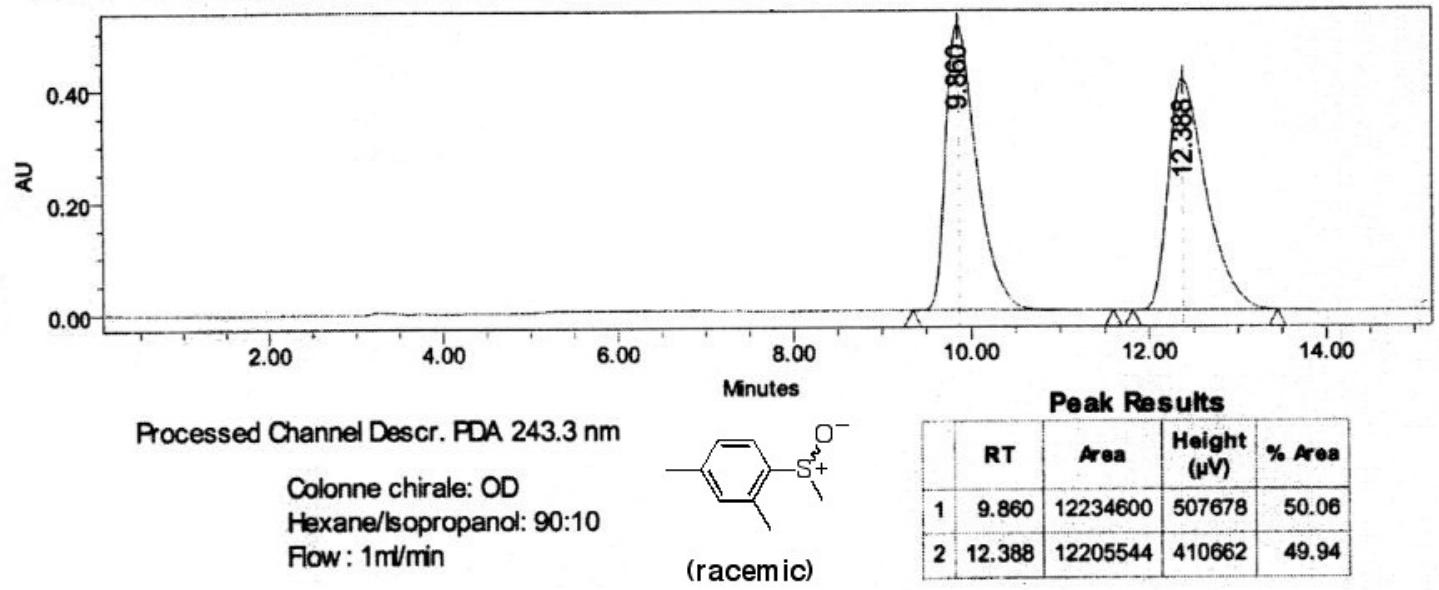



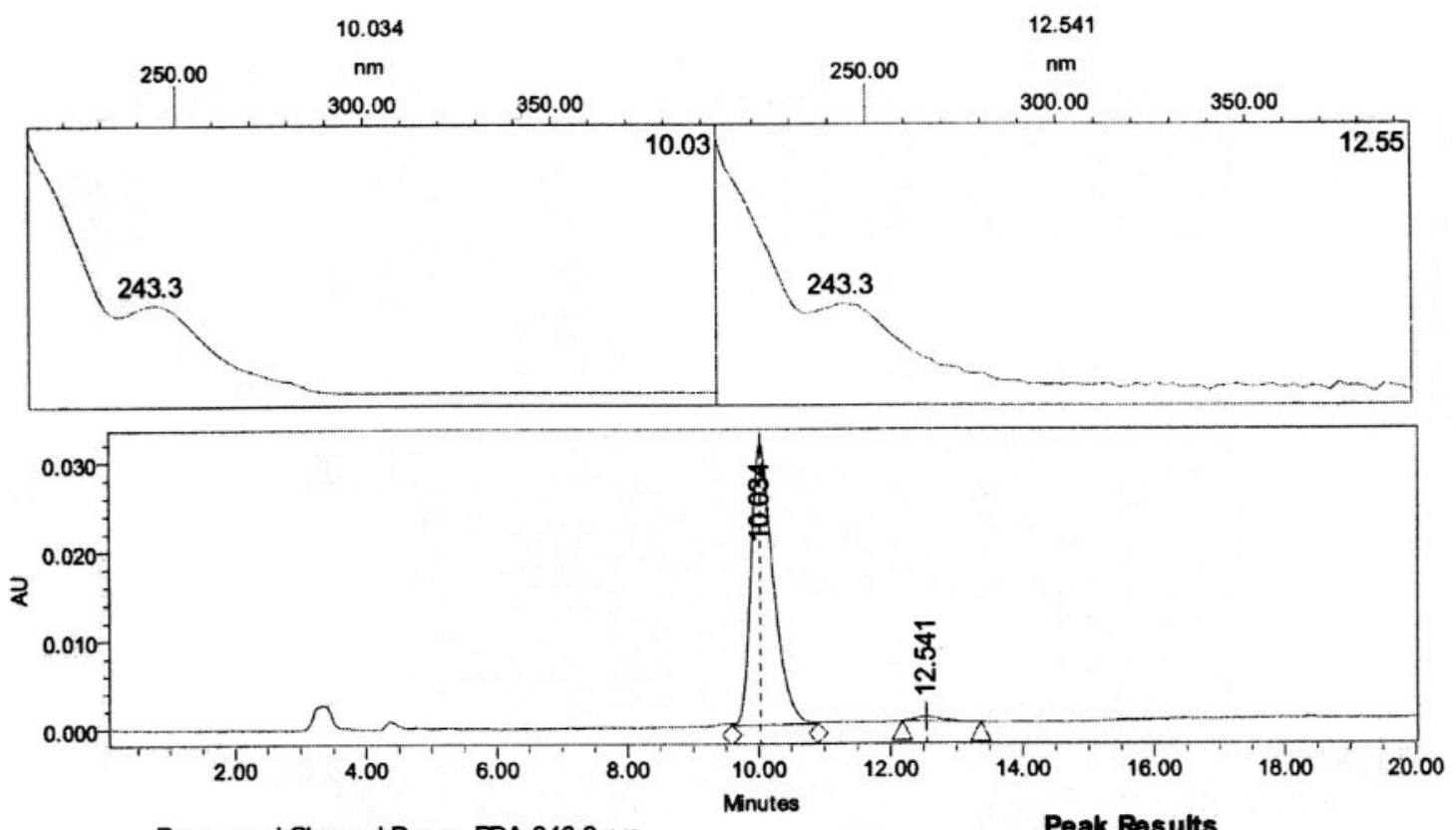

Processed Channel Descr. PDA $243.3 \mathrm{~nm}$

Colonne chirale: $O D$

Hexane/lsopropanol: 90:10

Flow: $1 \mathrm{~m} / \mathrm{min}$

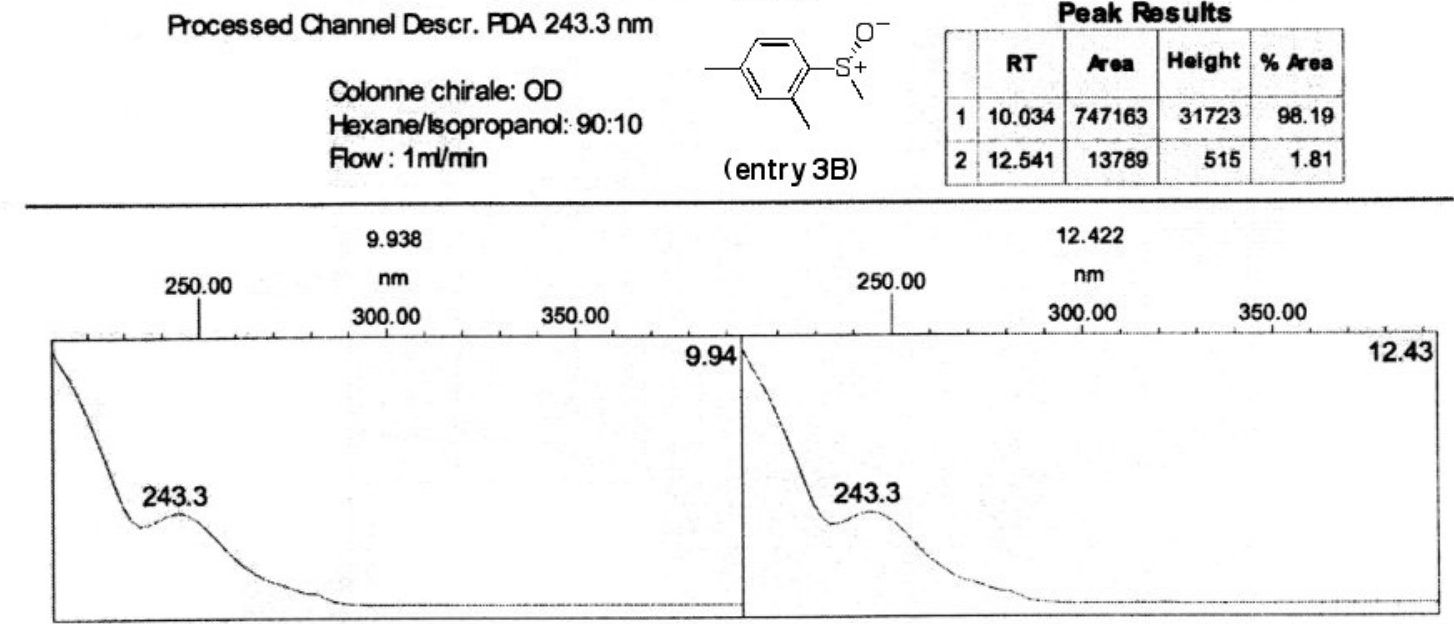

Peak Results

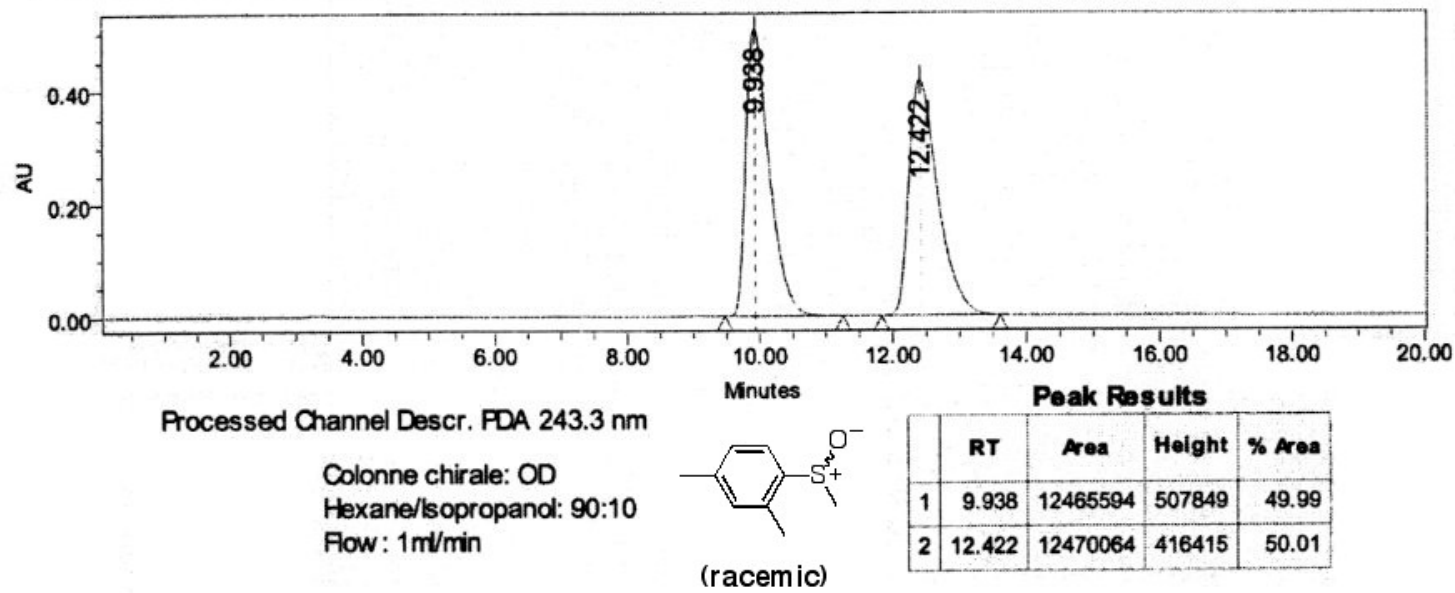



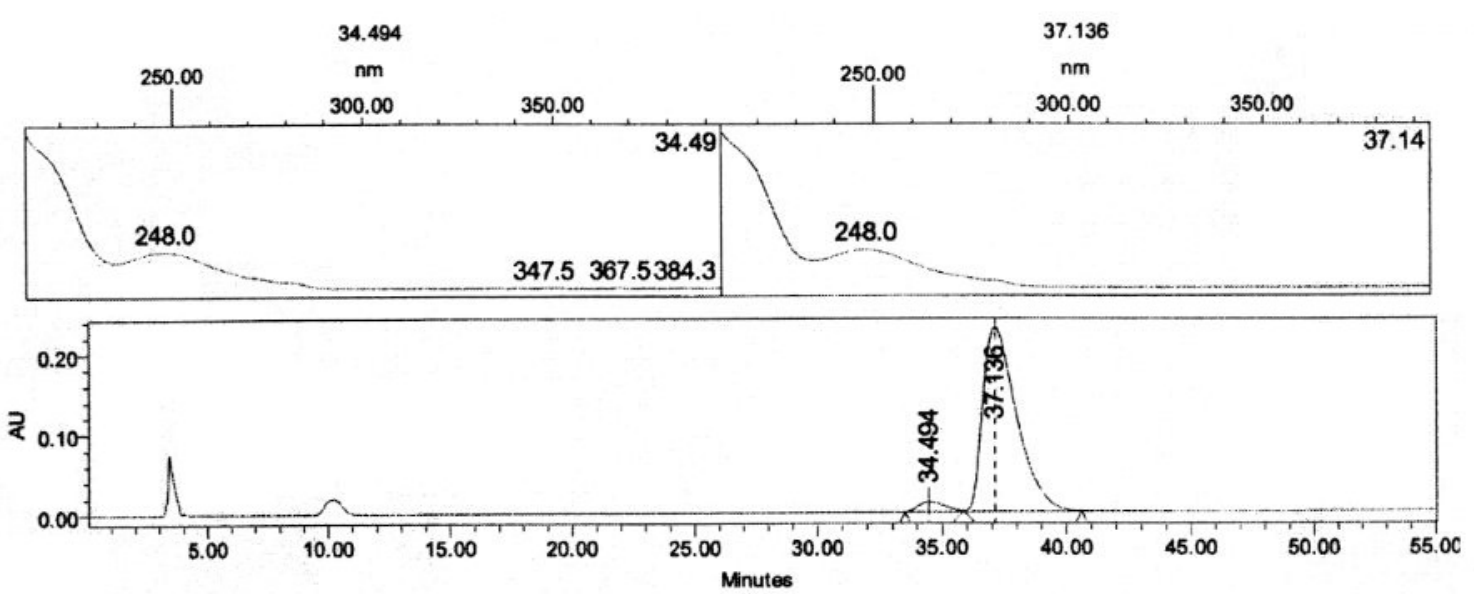

Processed Channel Descr. PDA MaxPlot (210.0 nm to $400.0 \mathrm{~nm}$ )

Colonne chirale: $\mathrm{OD}$

Hexane/lsopropanol: 98.5: 1.5

Flow: $1 \mathrm{~m} / \mathrm{min}$

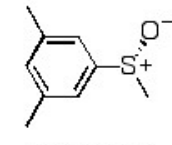

(entry 4A)

\begin{tabular}{|l|c|r|r|}
\hline & RT & \multicolumn{1}{|c|}{ Area } & \% Area \\
\hline 1 & 37.136 & 21657379 & 95.83 \\
\hline 2 & 34.494 & 942483 & 4.17 \\
\hline
\end{tabular}
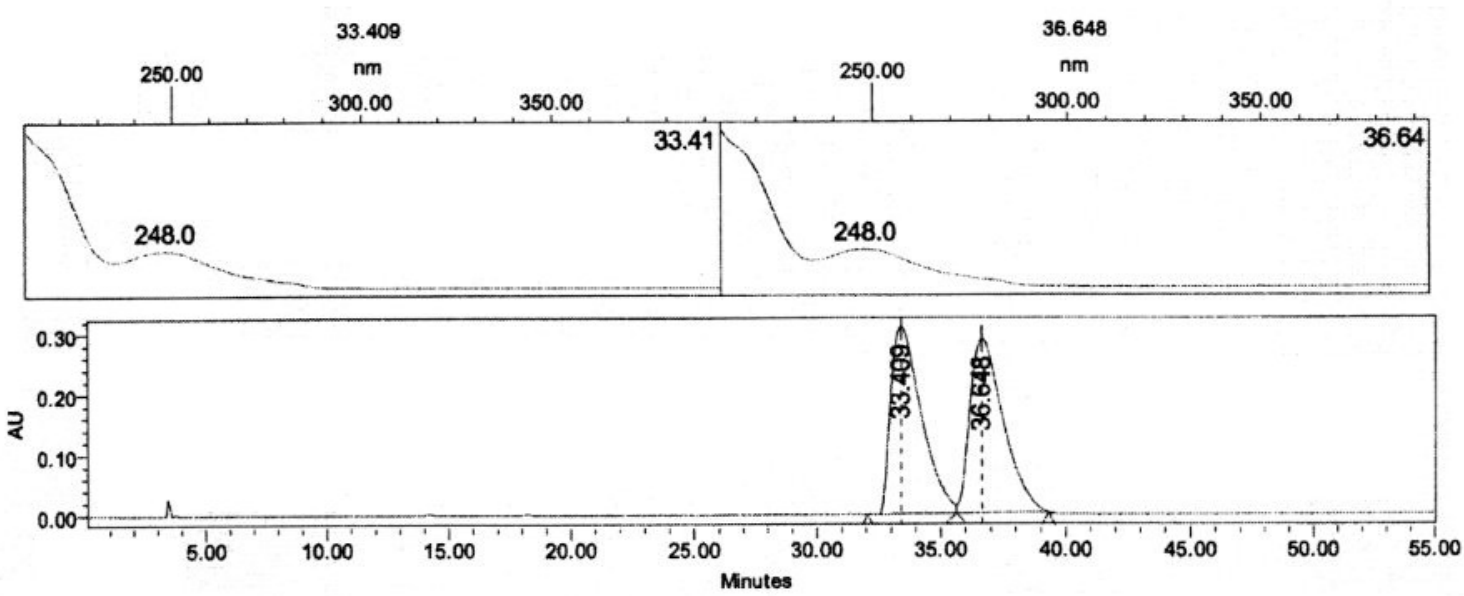

Processed Channel Descr. PDA MaxPlot (210.0 nm to $400.0 \mathrm{~nm})$

Colonne chirale: $O D$

Hexane/sopropanol: 98.5: 1.5

Flow: $1 \mathrm{~m} / \mathrm{min}$

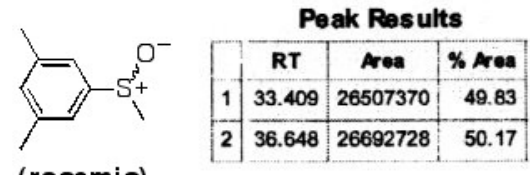

(racemic) 

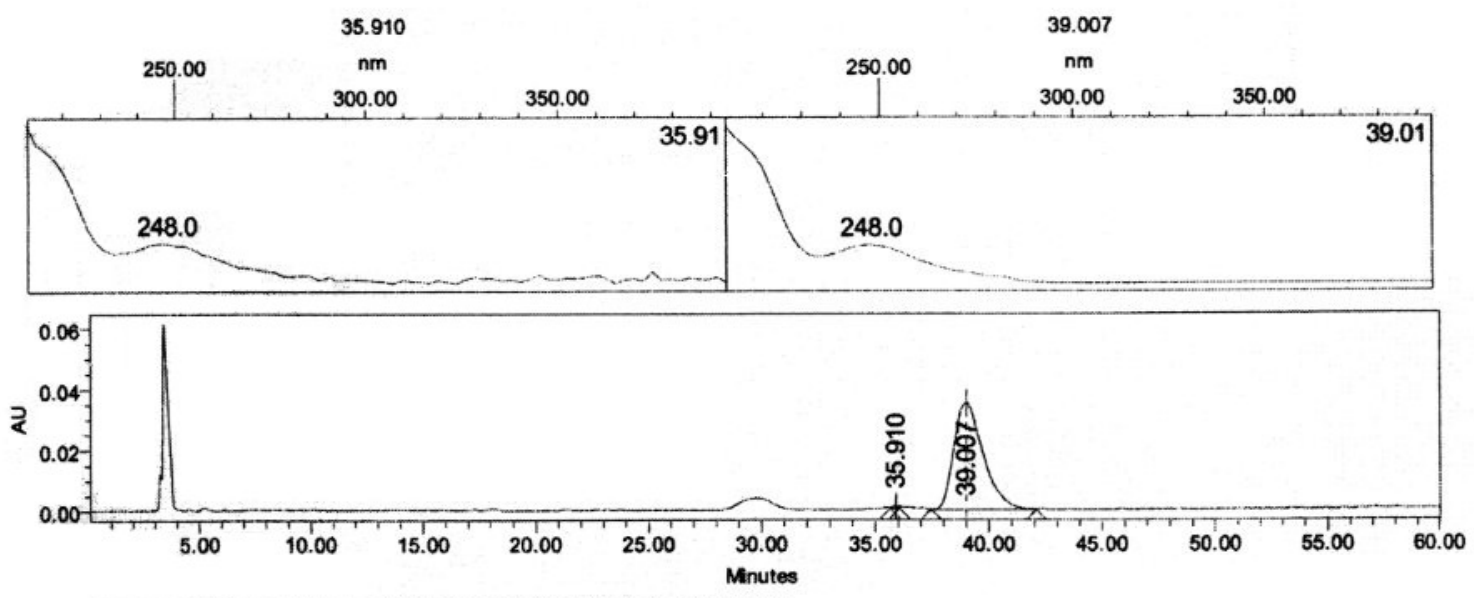

Processed Channel Descr. PDA MaxPlot (210.0 nm to $400.0 \mathrm{~nm}$ )

Colonne chirale: $O D$

Hexane/sopropanol: 98.5: 1.5

How: $1 \mathrm{~m} / \mathrm{min}$

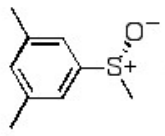

Peak Results

(entry 4B)

\begin{tabular}{|c|c|c|r|}
\hline & RT & \multicolumn{1}{|c|}{ Area } & \% Area \\
\hline 1 & 35.910 & 15789 & 0.50 \\
\hline 2 & 39.007 & 3168463 & 99.50 \\
\hline
\end{tabular}
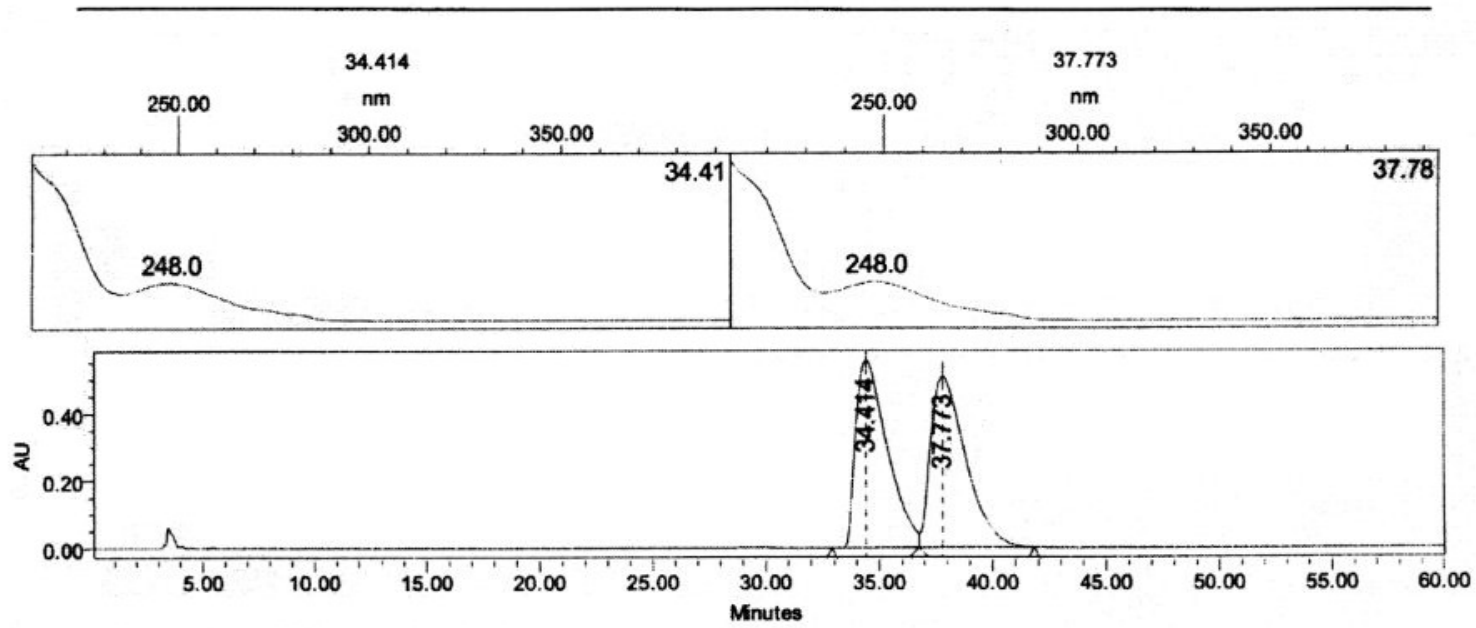

Processed Channel Descr. PDA MaxPlot (210.0 nm to $400.0 \mathrm{~nm})$

Colonne chirale: $O D$

Hexane/sopropanol: 98.5: 1.5

Fow : $1 \mathrm{~m} 1 / \mathrm{min}$

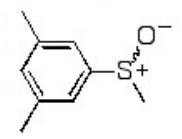

(racemic)
Peak Results

\begin{tabular}{|c|c|c|c|}
\hline & RT & Area & \% Area \\
\hline 1 & 34.414 & 53289696 & 49.52 \\
\hline 2 & 37.773 & 54313528 & 50.48 \\
\hline
\end{tabular}



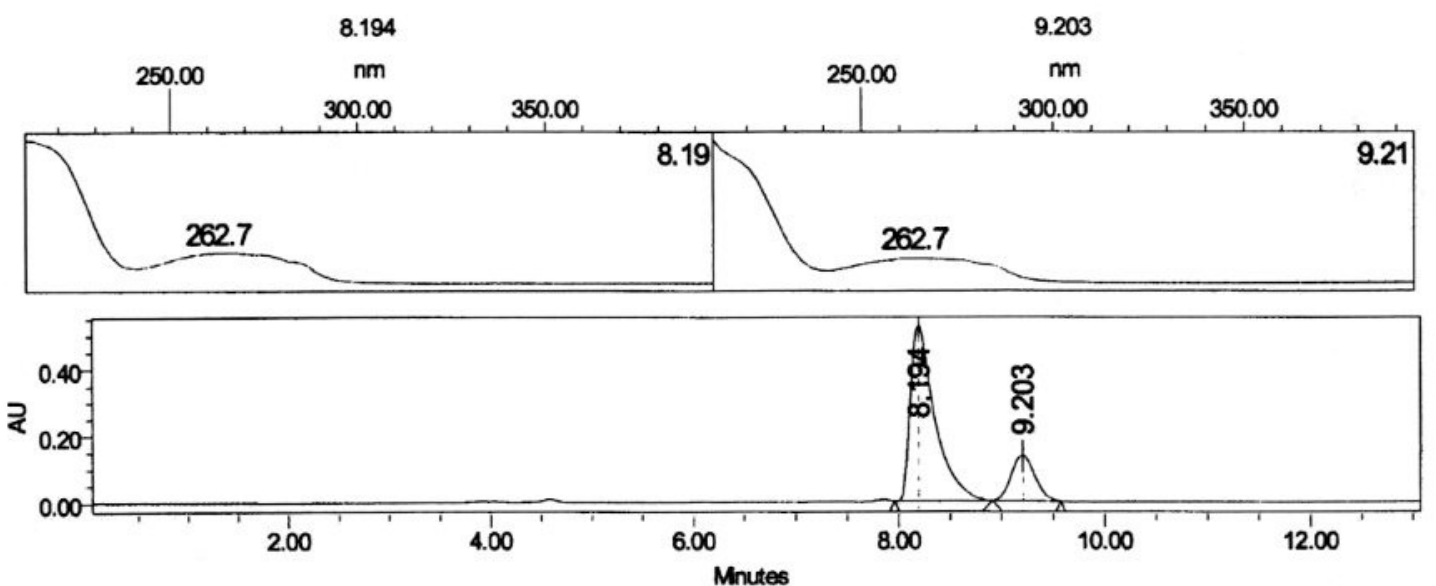

Colome: OD

Hexane/sopropend : 90/10

Fow. $0.8 \mathrm{~m} / \mathrm{min}$

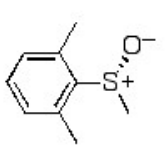

Proc. Orv. Descr.: PDA $230.0 \mathrm{~mm}$

(entry 5)

Peak Results
\begin{tabular}{|c|c|c|c|r|}
\hline & RT & Area & Height & $\%$ Area \\
\hline 1 & 8.194 & 8651142 & 525945 & 79.93 \\
\hline 2 & 9.203 & 2172030 & 135714 & 20.07 \\
\hline
\end{tabular}
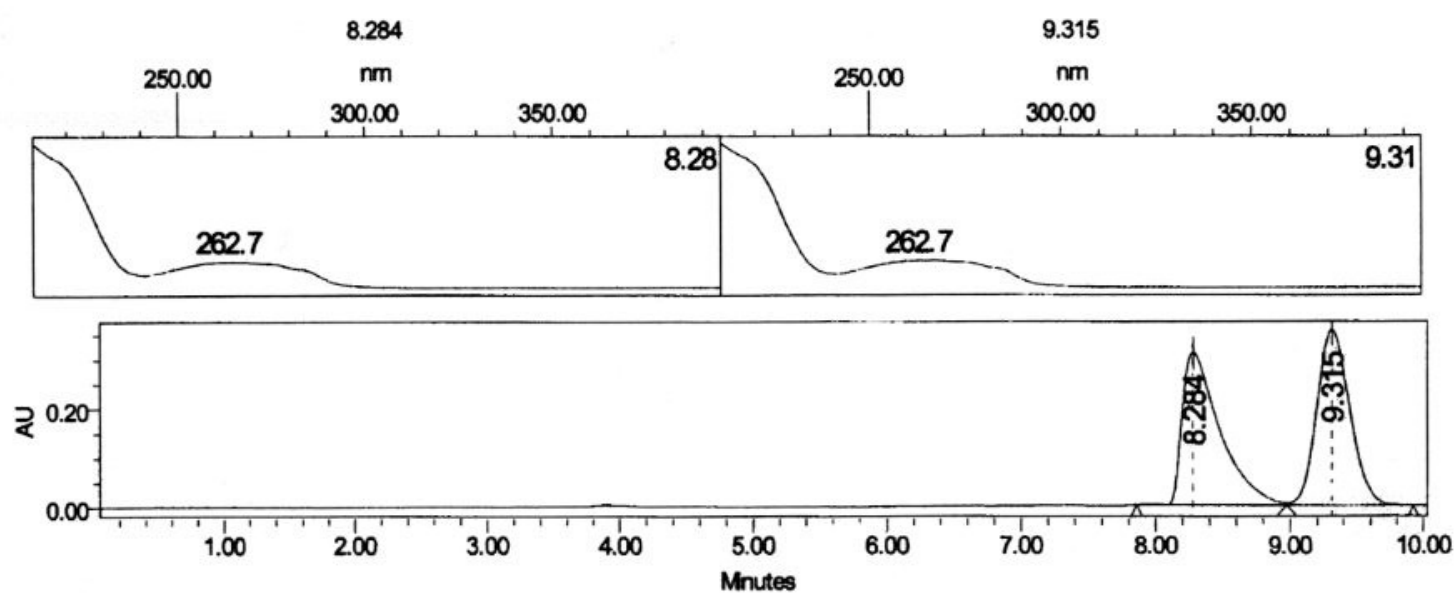

Colonne: OD

Hexane/sopropend: $90 / 10$

Flow. $0.8 \mathrm{~m} / \mathrm{min}$

Proc. Crrl. Descr.: PDA $230.0 \mathrm{~mm}$

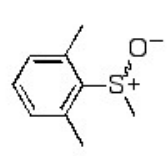

(racemic)

Peak Results
\begin{tabular}{|c|c|c|c|r|}
\hline & RT & Area & Height & $\%$ Area \\
\hline 1 & 8.284 & 6016354 & 309832 & 49.64 \\
\hline 2 & 9.315 & 6104669 & 356369 & 50.36 \\
\hline
\end{tabular}



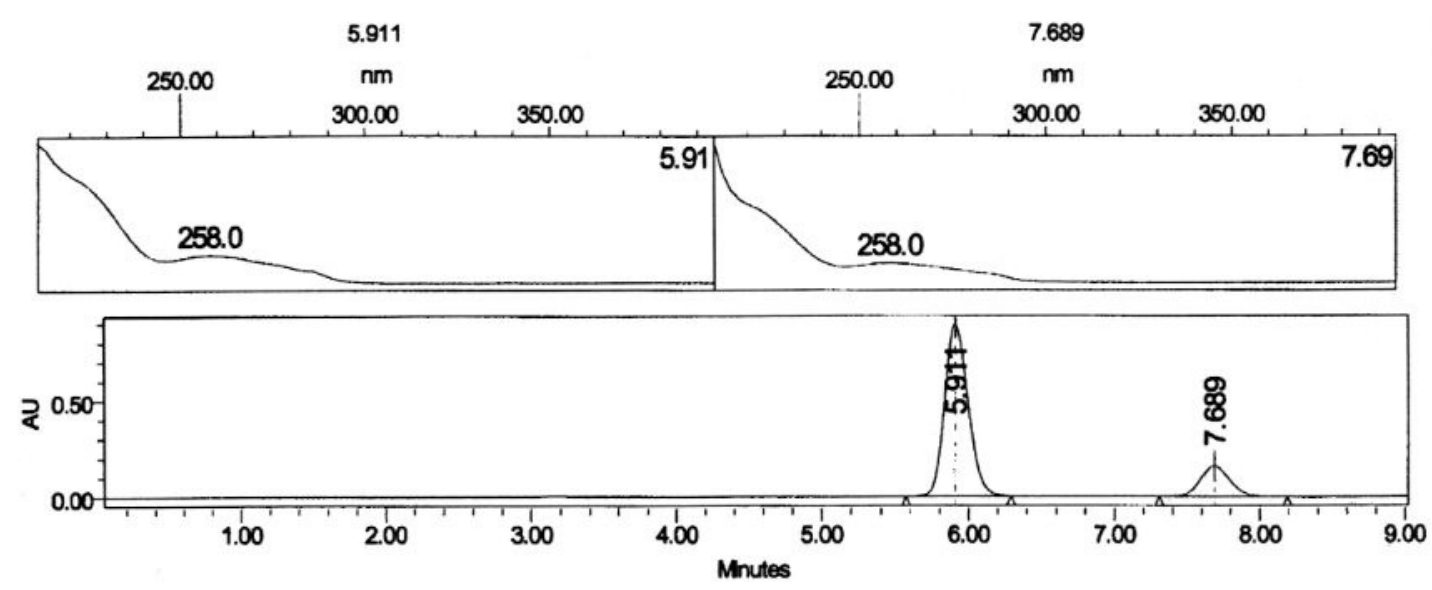

Colone: $\infty$

Hexene/lsopropend : 90/10

Fow. $1 \mathrm{~m} / \mathrm{min}$

Proc. Crr. Descr.: PDA $230.0 \mathrm{~mm}$

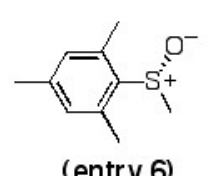

Peak Results

\begin{tabular}{|c|c|c|c|r|}
\hline & RI & Area & Height & \% Area \\
\hline 1 & 5.911 & 9977954 & 895361 & 81.83 \\
\hline 2 & 7.689 & 2215313 & 157134 & 18.17 \\
\hline
\end{tabular}
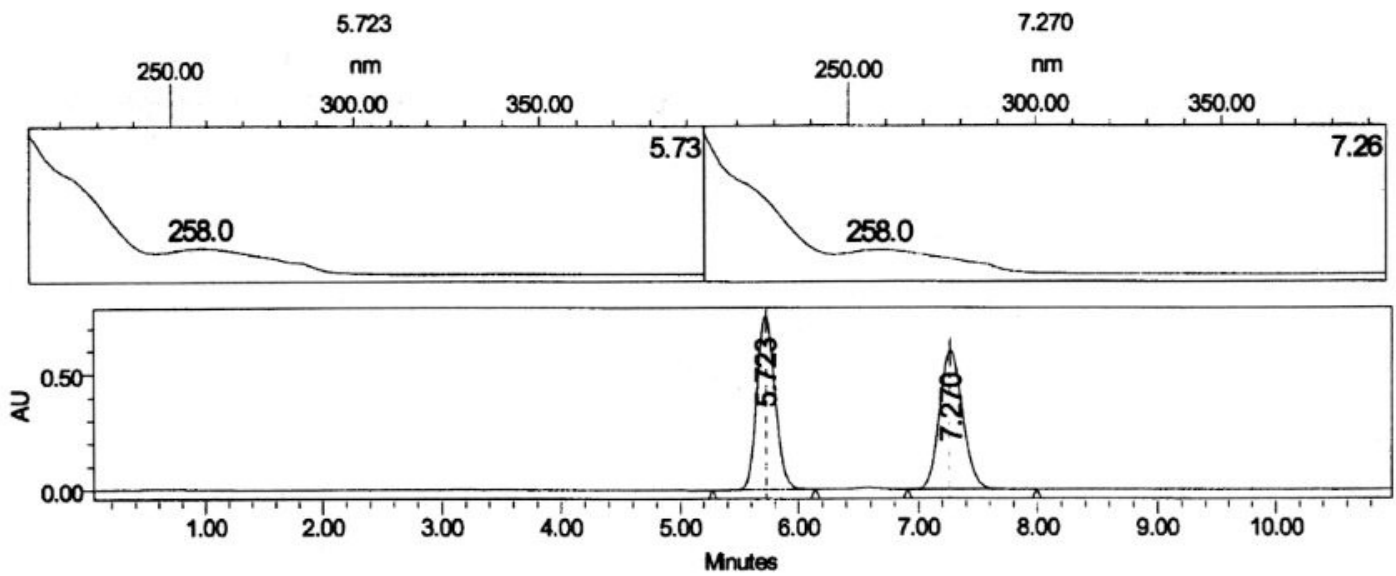

Colonne: OD

Hexane/sopropand: : $90 / 10$

Fow. $1 \mathrm{~m} / \mathrm{min}$

Proc. Orw. Descr.: PDA $230.0 \mathrm{~mm}$

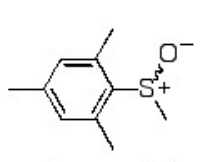

(racemic)

Peak Results
\begin{tabular}{|c|c|c|c|r|}
\hline & RT & Area & Height & $\%$ Area \\
\hline 1 & 5.723 & 7985159 & 754967 & 49.83 \\
\hline 2 & 7.270 & 8040938 & 601352 & 50.17 \\
\hline
\end{tabular}



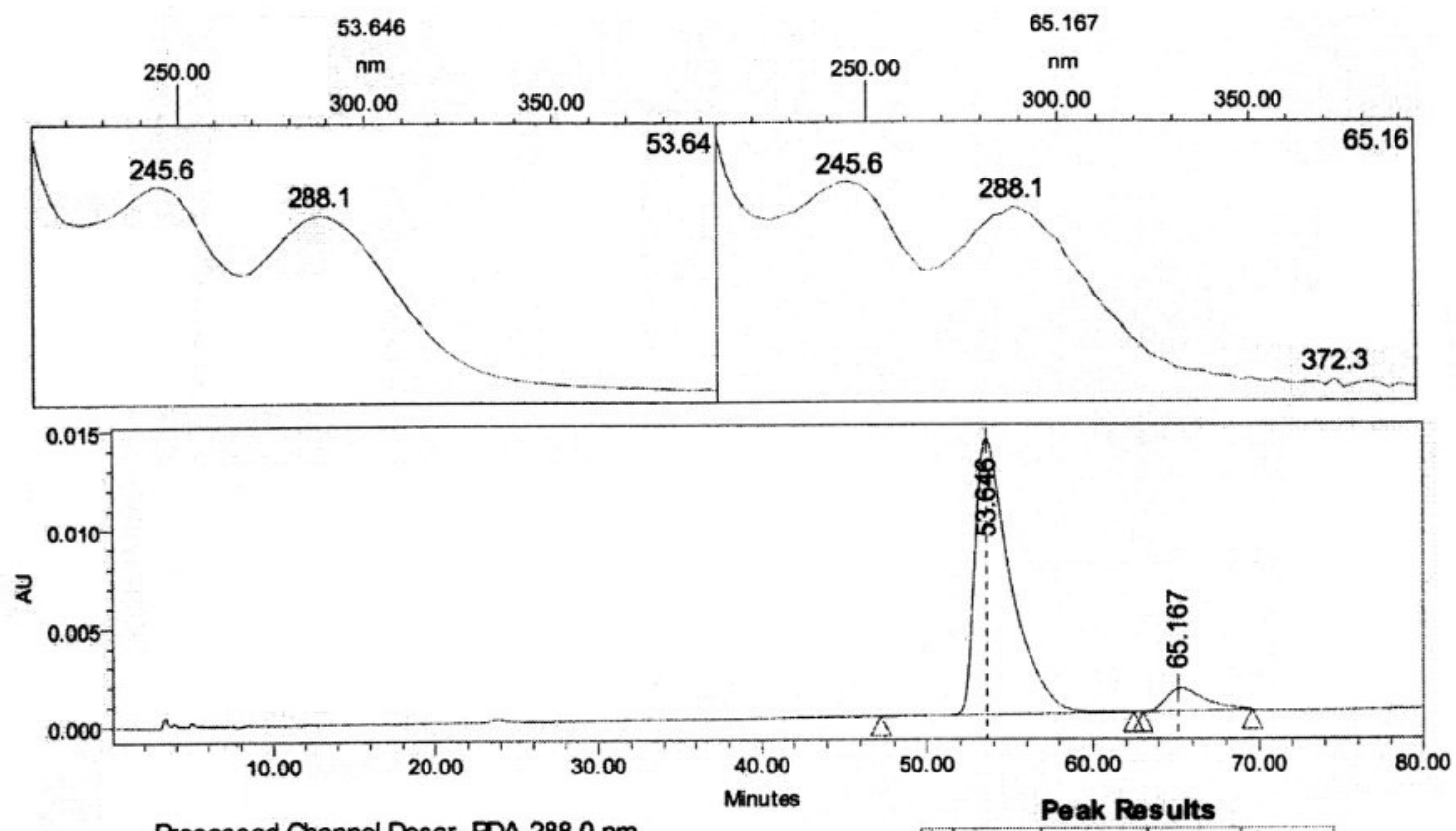

Processed Channel Descr. PDA $288.0 \mathrm{~nm}$

Colonne chirale: OS

Hexane/lsopropanol: 90:10

Fow: $1 \mathrm{~m} / \mathrm{min}$
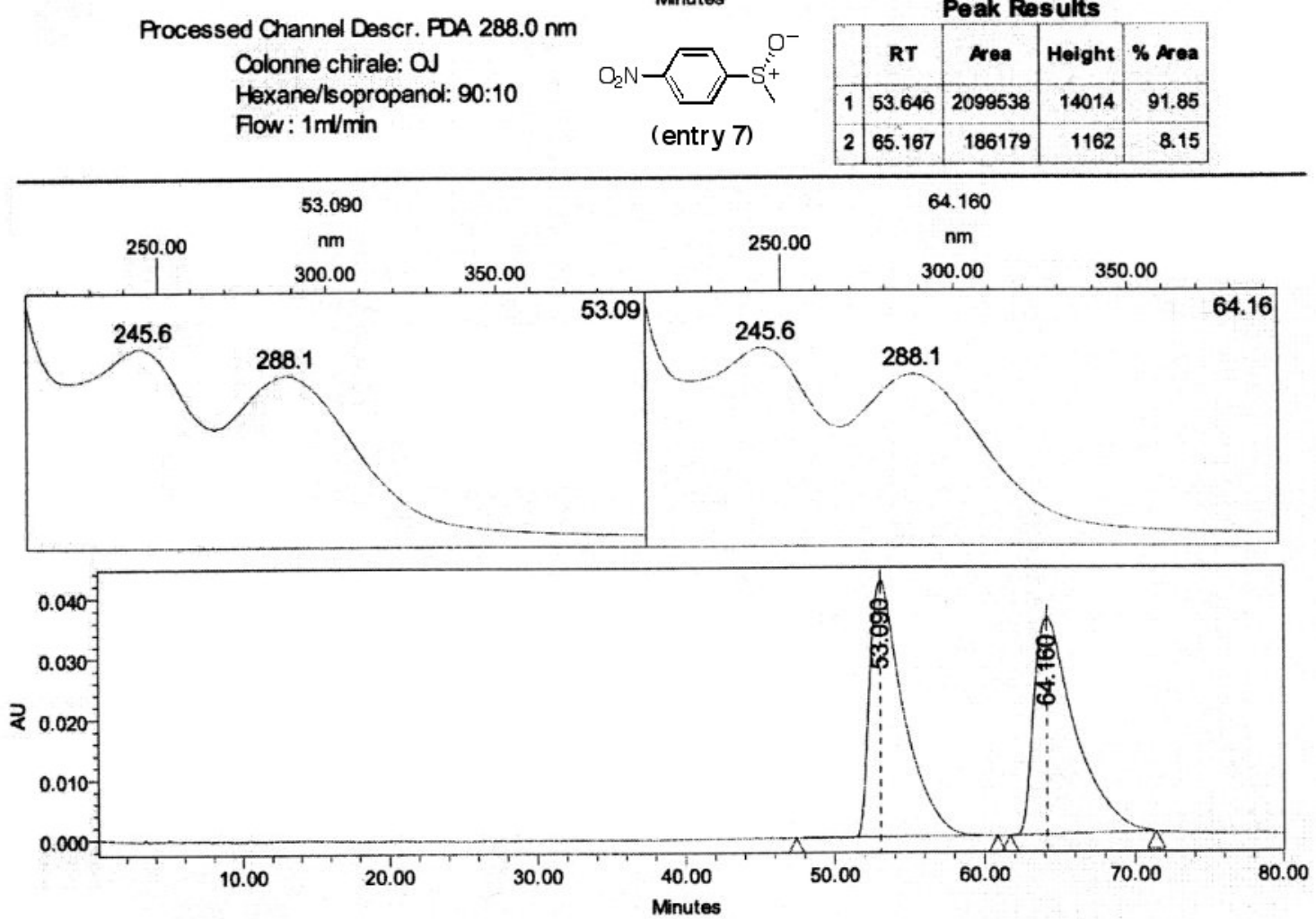

Processed Channel Descr. PDA $288.0 \mathrm{~nm}$

Peak Results

Colonne chirale: OS

Hexane/sopropanol: 90:10

Flow: $1 \mathrm{~m} / / \mathrm{min}$

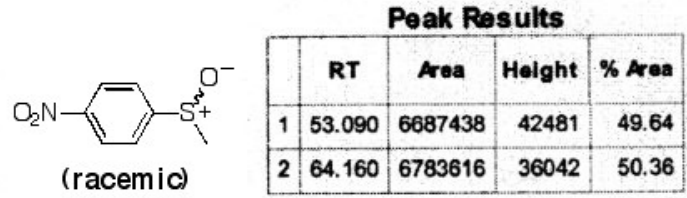



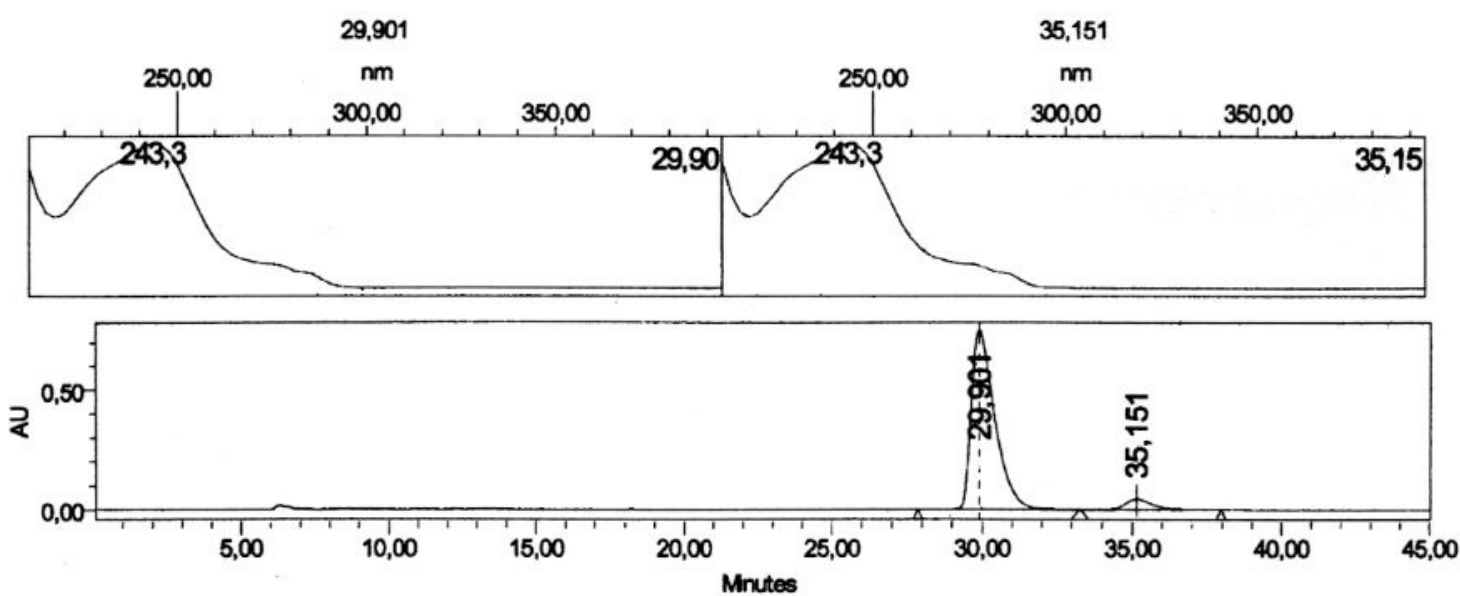

Colorne: OD

Hexane/isopropand : $90 / 10$

Flow. 0,5ml/min

Proc. Chr. Descr.: PDA 220,0 nm

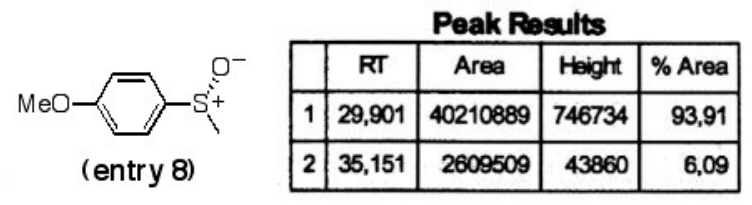
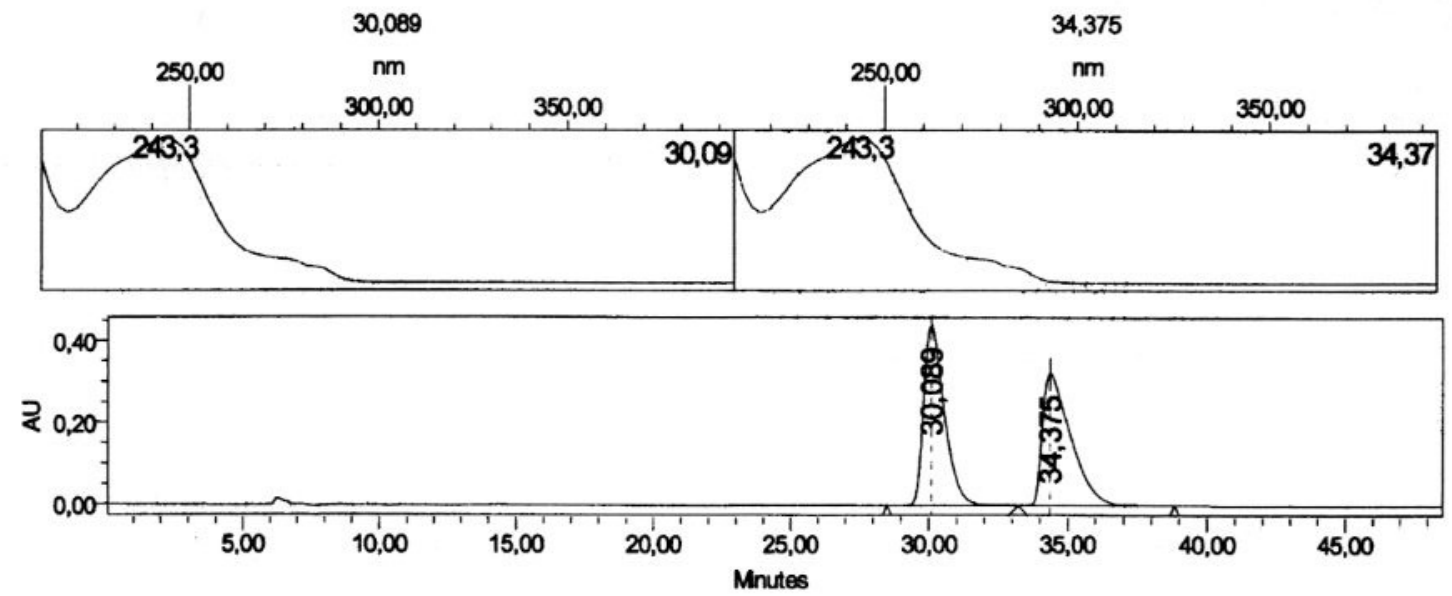

Colorne: OD

Hexane/isopropend: :90/10

Flow. 0,5 $\mathrm{m} / \mathrm{min}$

Proc. Onl. Descr.: PDA 220,0 nm

\begin{tabular}{|c|c|c|c|c|c|}
\cline { 3 - 6 } & & RT & Area & Height & \% Area \\
\cline { 2 - 6 } & 1 & 30,089 & 22527938 & 437230 & 49,94 \\
\cline { 2 - 6 } & 2 & 34,375 & 22585077 & 320575 & 50,06 \\
\hline
\end{tabular}



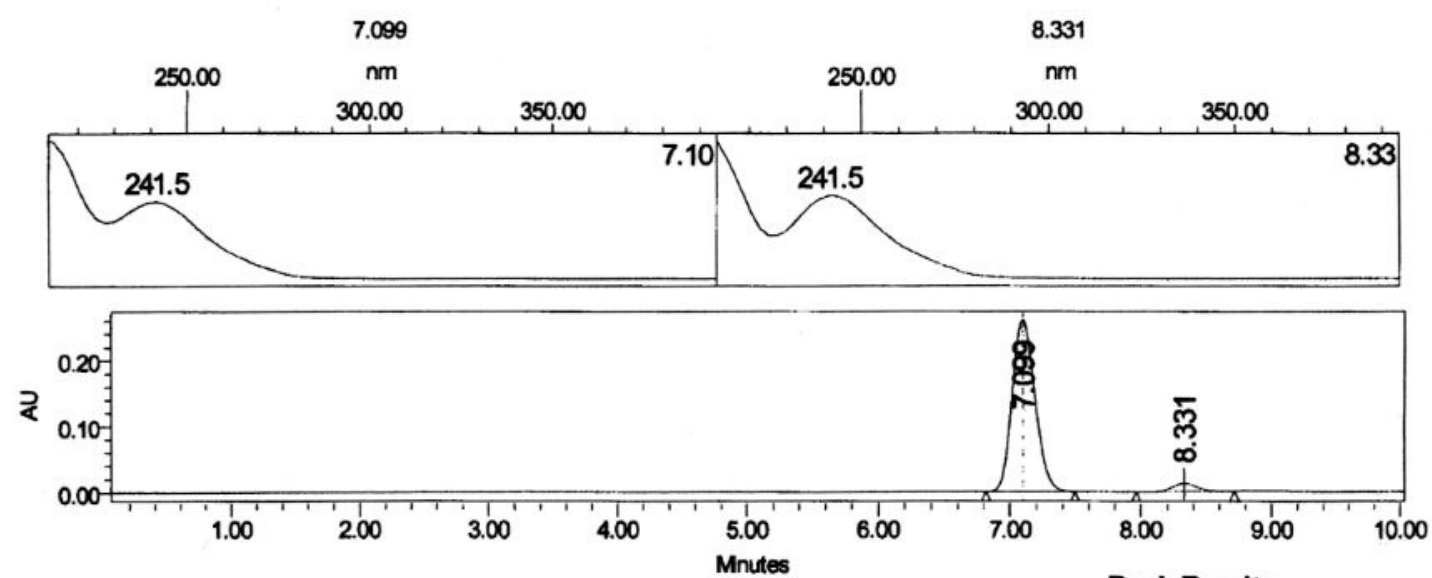

Colonne: OD

Hexane/sopropand : 90/10

Fow. $1 \mathrm{~m} / \mathrm{min}$

Proc. Onl. Descr.: PDA $230.0 \mathrm{~mm}$

\begin{tabular}{l|c|c|c|c|r|}
\hline & & \multicolumn{5}{|c|}{ Peak Results } \\
\cline { 2 - 6 } & & RT & Area & Height & \% Area \\
\cline { 2 - 6 } & 1 & 7.099 & 3115645 & 260449 & 95.03 \\
\hline (entry 9) & 2 & 8.331 & 163065 & 12530 & 4.97 \\
\hline
\end{tabular}
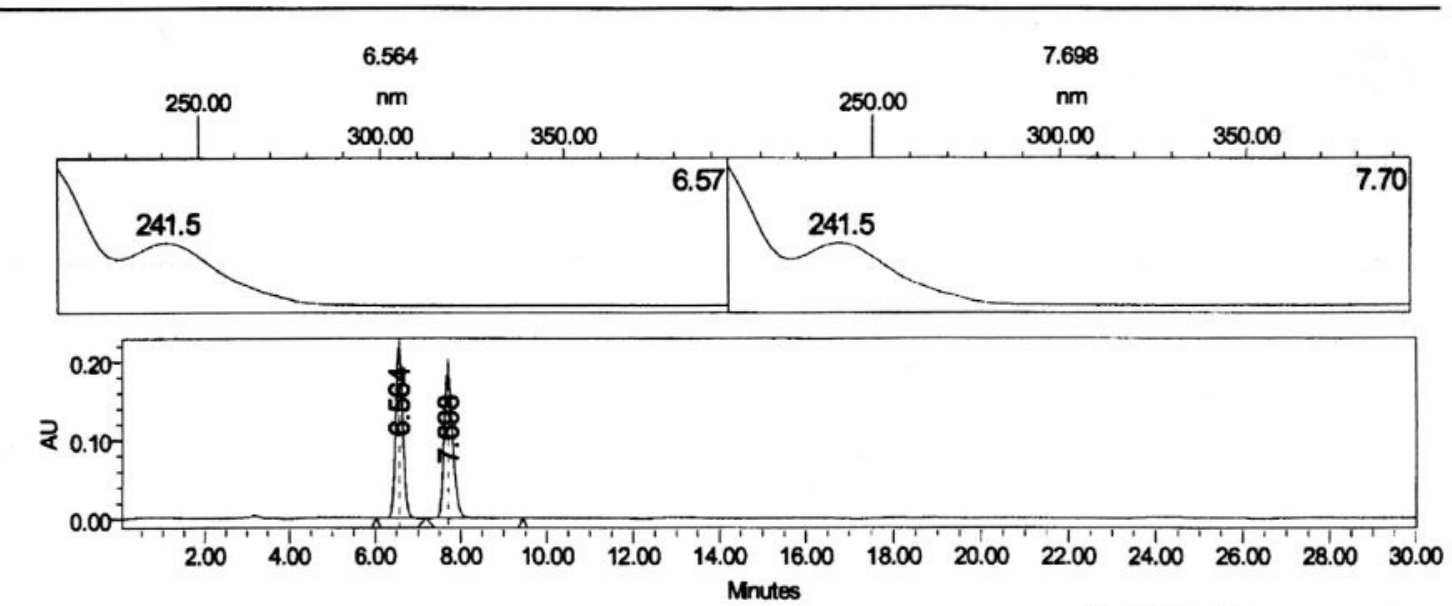

Colonne: OD

Hexene/lsopropand: : 90/10

Fow. $1 \mathrm{~m} / \mathrm{min}$

Proc. Onl. Descr.: PDA $230.0 \mathrm{~mm}$

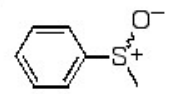

(racemic)
Peak Results

\begin{tabular}{|c|c|c|c|r|}
\hline & RI & Area & Height & \% Area \\
\hline 1 & 6.564 & 2558978 & 218370 & 49.37 \\
\hline 2 & 7.698 & 2624584 & 183116 & 50.63 \\
\hline
\end{tabular}



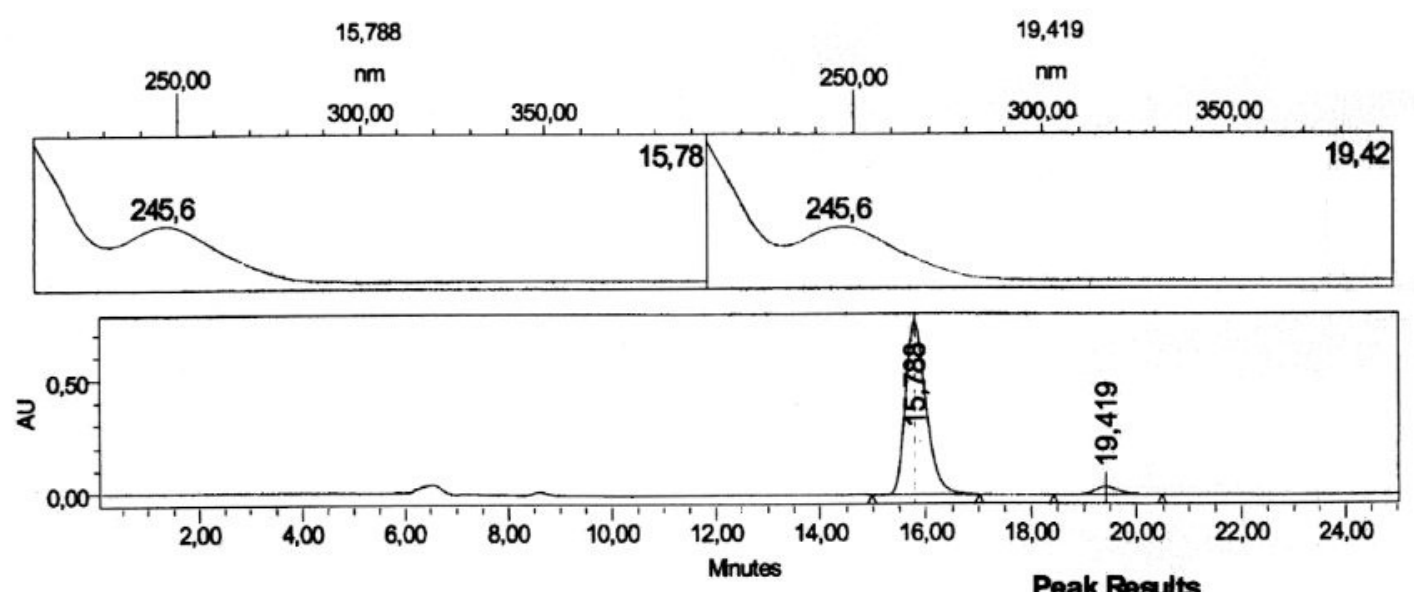

Colonne: OD

Hexane/isopropand :90/10

Flow. 0,5ml/min

Proc. Onl. Descr.: PDA 220,0 mm (entry 10)

\begin{tabular}{|c|c|c|c|c|r|}
\cline { 3 - 6 } & & RT & Area & Height & \% Area \\
\hline (entry 10) & 1 & 15,788 & 20645050 & 762287 & 95,18 \\
\hline 2 & 19,419 & 1045566 & 33109 & 4,82 \\
\hline
\end{tabular}

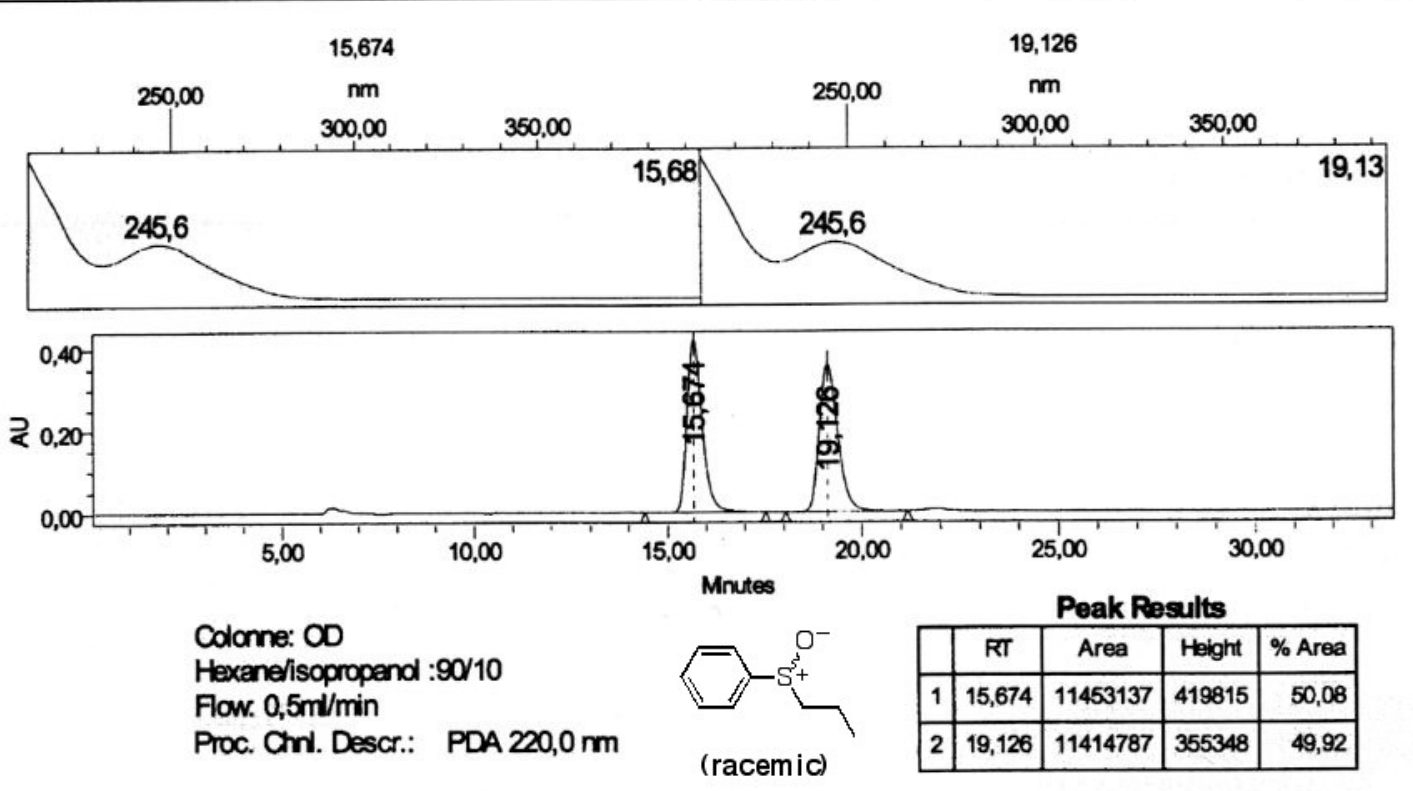



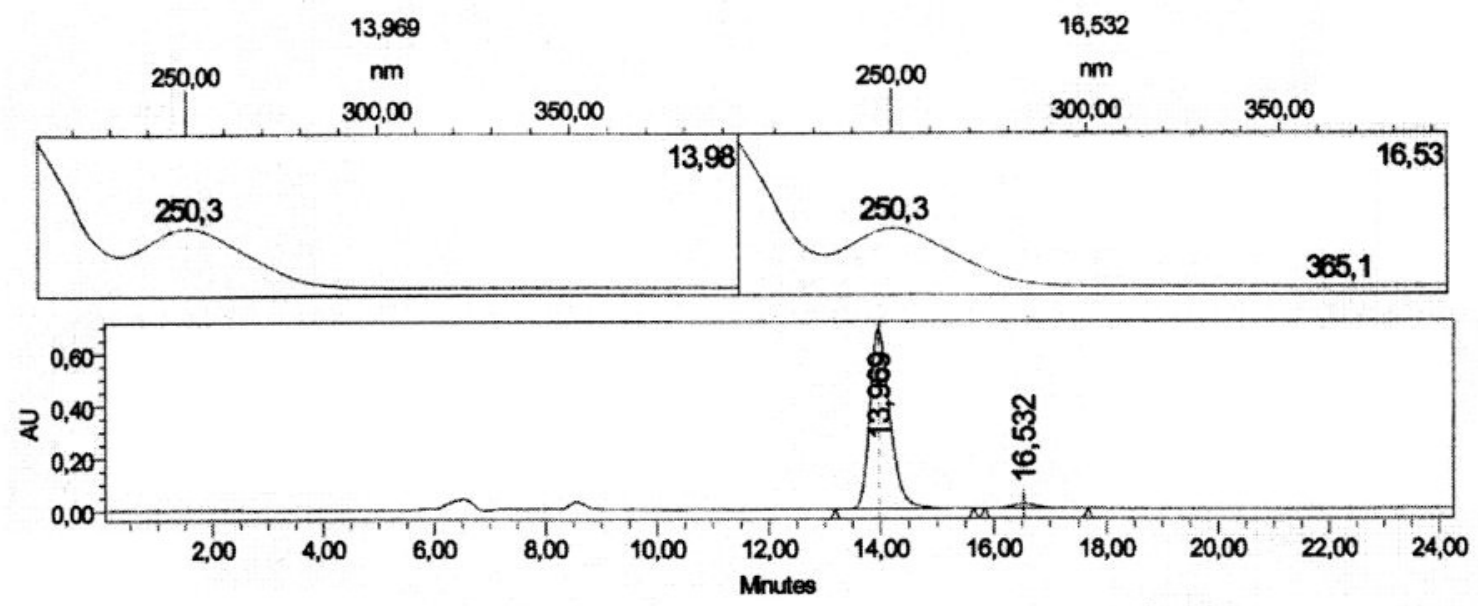

Colome: $O D$

Hexane/isopropand: :90/10

Flow. $0,5 \mathrm{~m} / \mathrm{min}$

Proc. Onnl. Descr:: PDA 220,0 mm

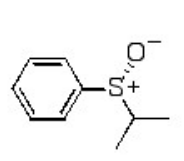

(entry 11)

Peak Results
\begin{tabular}{|r|c|r|r|r|}
\hline & \multicolumn{1}{|c|}{ RT } & \multicolumn{1}{|c|}{ Area } & Height & $\%$ Area \\
\hline 1 & 13,969 & 17068189 & 683315 & 97,13 \\
\hline 2 & 16,532 & 504470 & 18481 & 2,87 \\
\hline
\end{tabular}
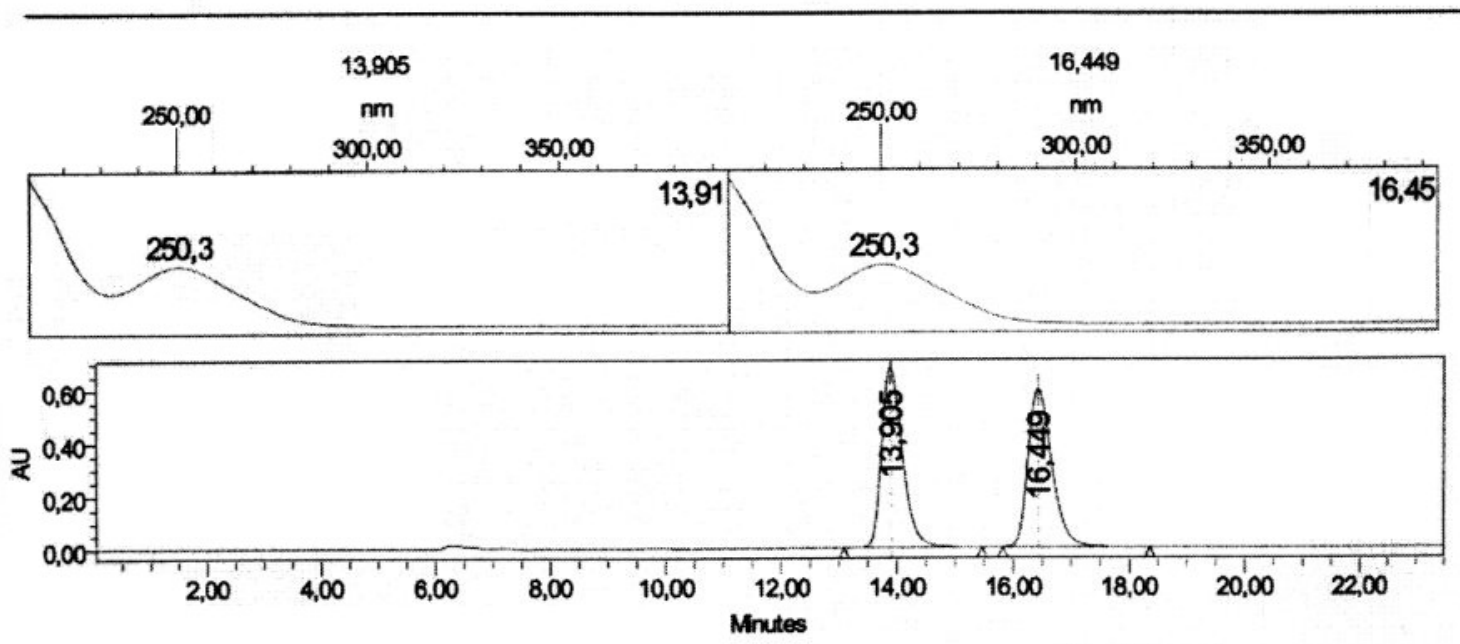

Colorne: $\infty$

Hexene/isopropend : $90 / 10$

Flow. 0,5m//min

Proc. Conv. Descr.: PDA 220,0 $\mathrm{mm}$

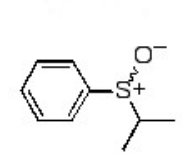

(racemic)

Peak Resslts
\begin{tabular}{|c|c|c|c|r|}
\hline & RT & Area & Height & $\%$ Area \\
\hline 1 & 13,905 & 16919926 & 677108 & 50,08 \\
\hline 2 & 16,449 & 16865588 & 592211 & 49,92 \\
\hline
\end{tabular}



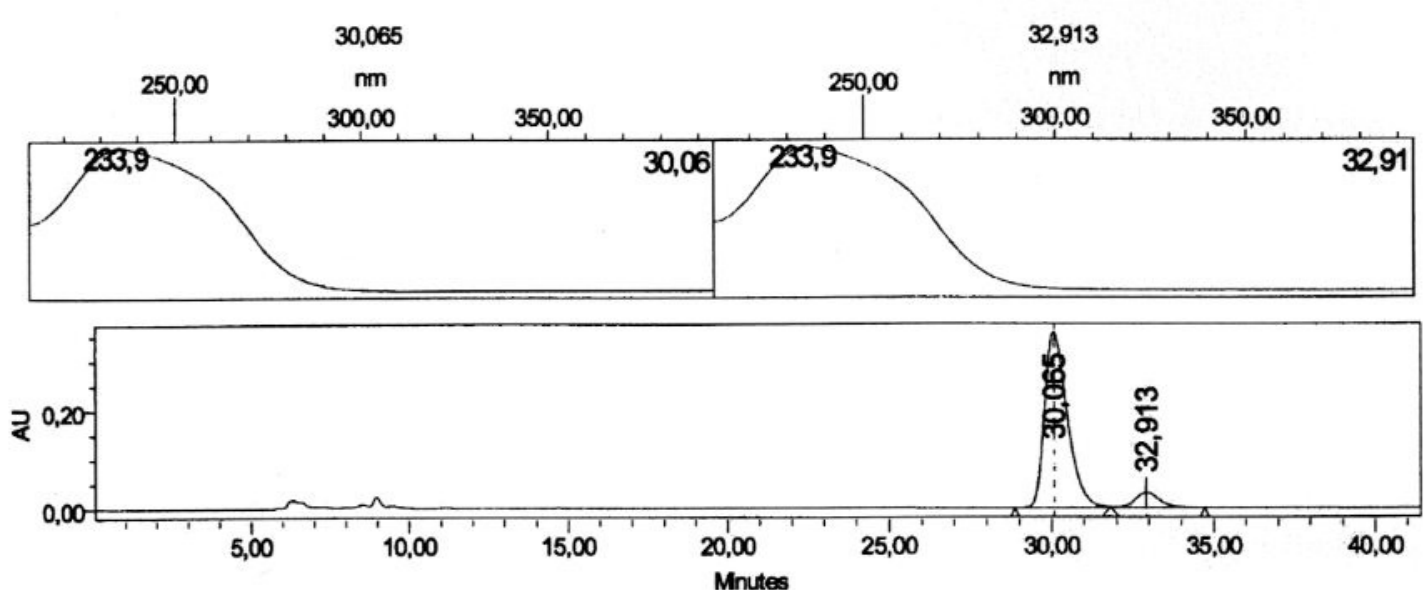

Colonne: $\infty$

Hexane/isopropand : 90/10

Flow. $0,5 \mathrm{~m} / \mathrm{min}$

Proc. Ond. Descr.: PDA 220,0 nm

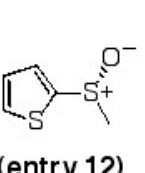

Peak Results

\begin{tabular}{|c|c|c|r|r|}
\hline & RT & Area & Height & \% Area \\
\hline 1 & 30,065 & 17256378 & 356726 & 91,85 \\
\hline 2 & 32,913 & 1531048 & 29532 & 8,15 \\
\hline
\end{tabular}
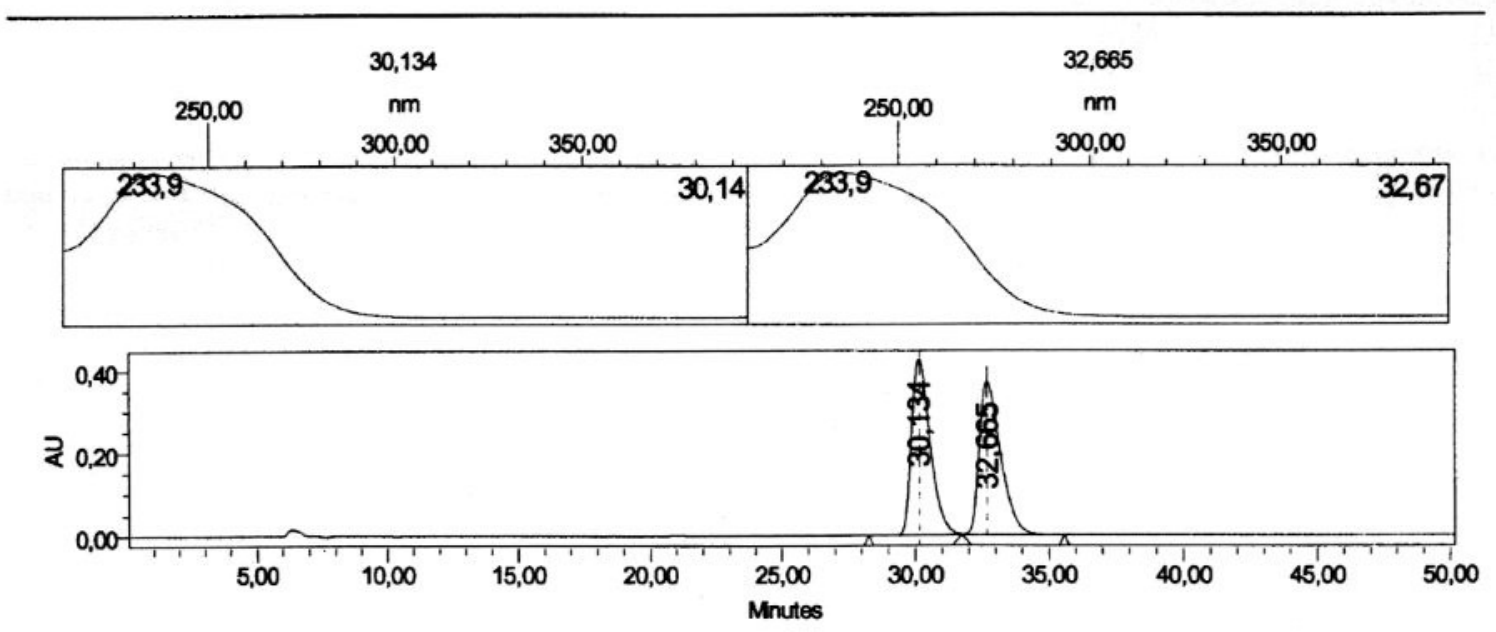

Colorne: OD

Hexane/isopropand :90/10

Fow. 0,5m//min

Proc. Onl. Descr.: PDA 220,0 nm

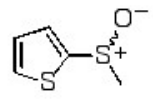

(racemic)

Peak Results

\begin{tabular}{|c|c|c|c|r|}
\hline & RT & Area & Height & \% Area \\
\hline 1 & 30,134 & 20556891 & 425464 & 49,96 \\
\hline 2 & 32,665 & 20590041 & 370860 & 50,04 \\
\hline
\end{tabular}



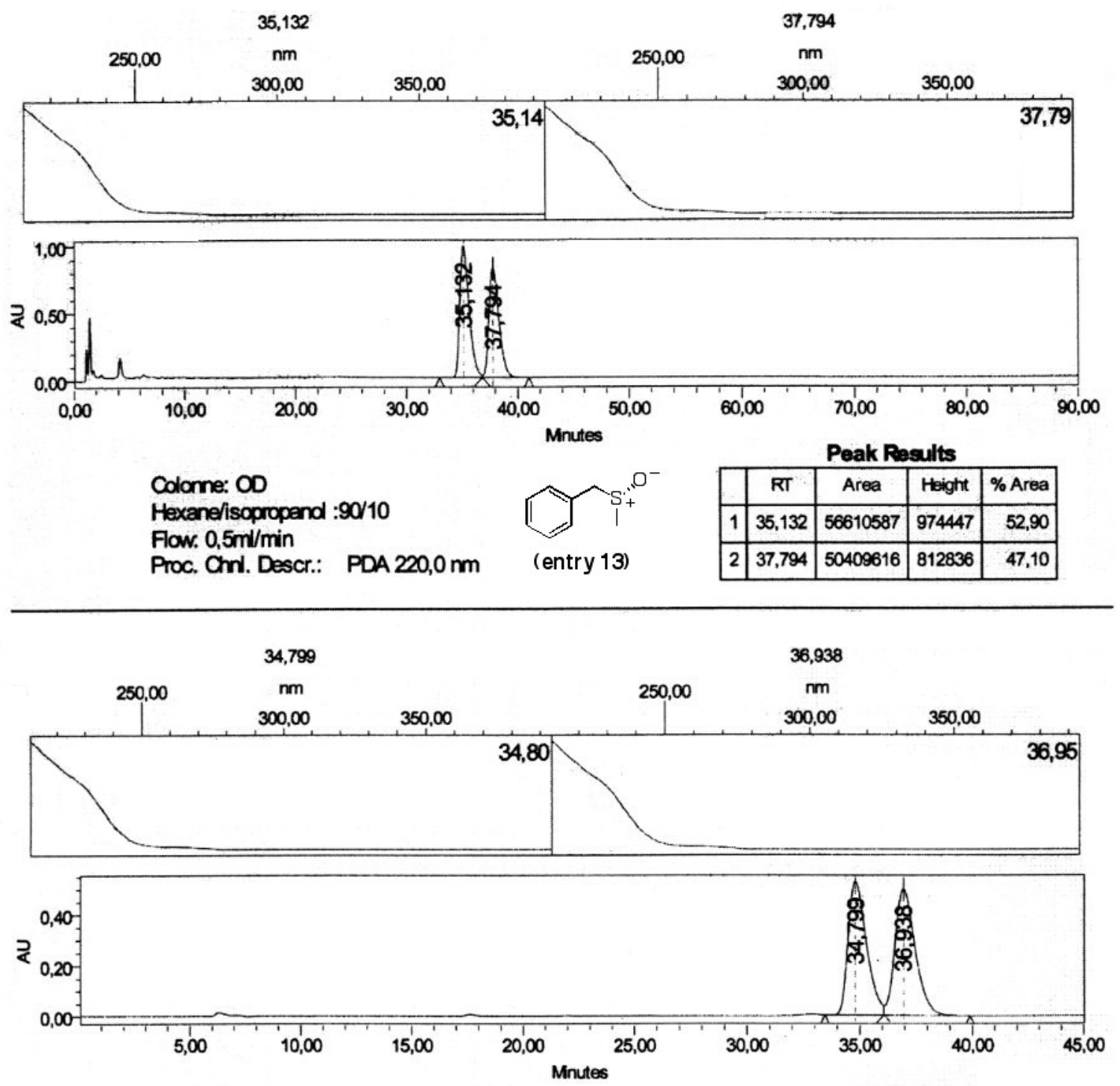

Colonne: OD

Hexane/iscpropand :90/10

Flow, 0,5m//min

Proc. Cnnl. Descr.: PDA 220,0 rm

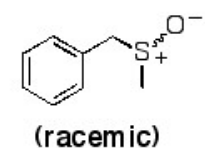

Peak Results

\begin{tabular}{|c|c|c|c|r|}
\hline & RT & Area & Height & \% Area \\
\hline 1 & 34,799 & 30180916 & 527348 & 49,22 \\
\hline 2 & 36,938 & 31137523 & 499116 & 50,78 \\
\hline
\end{tabular}



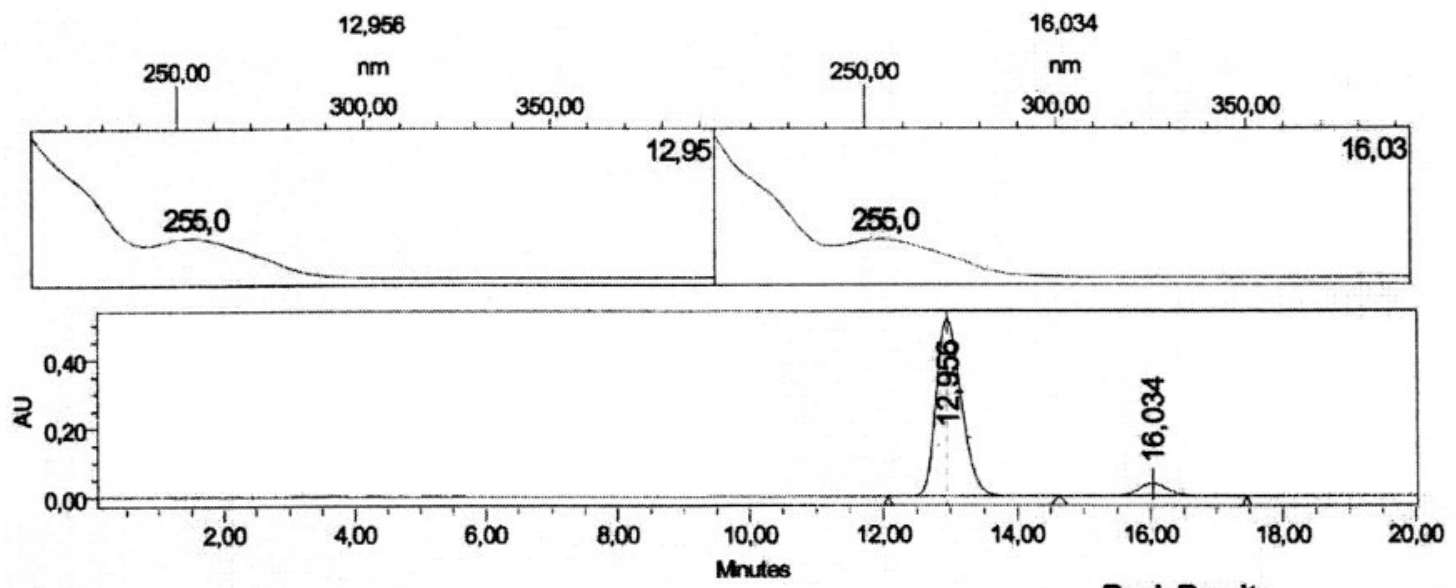

Colonne: $\infty$

Hexane/isopropand: :90/10

Fow. 1mV/min

Proc. Arl. Descr:: PDA 254,0 m

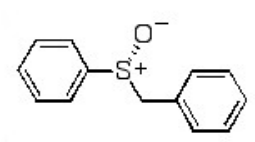

(entry 14)

Peak Results
\begin{tabular}{|r|c|c|r|r|}
\hline & Rा & Area & Height & $\%$ Area \\
\hline 1 & 12,956 & 13247301 & 515829 & 92,38 \\
\hline 2 & 16,034 & 1092230 & 35232 & 7,62 \\
\hline
\end{tabular}
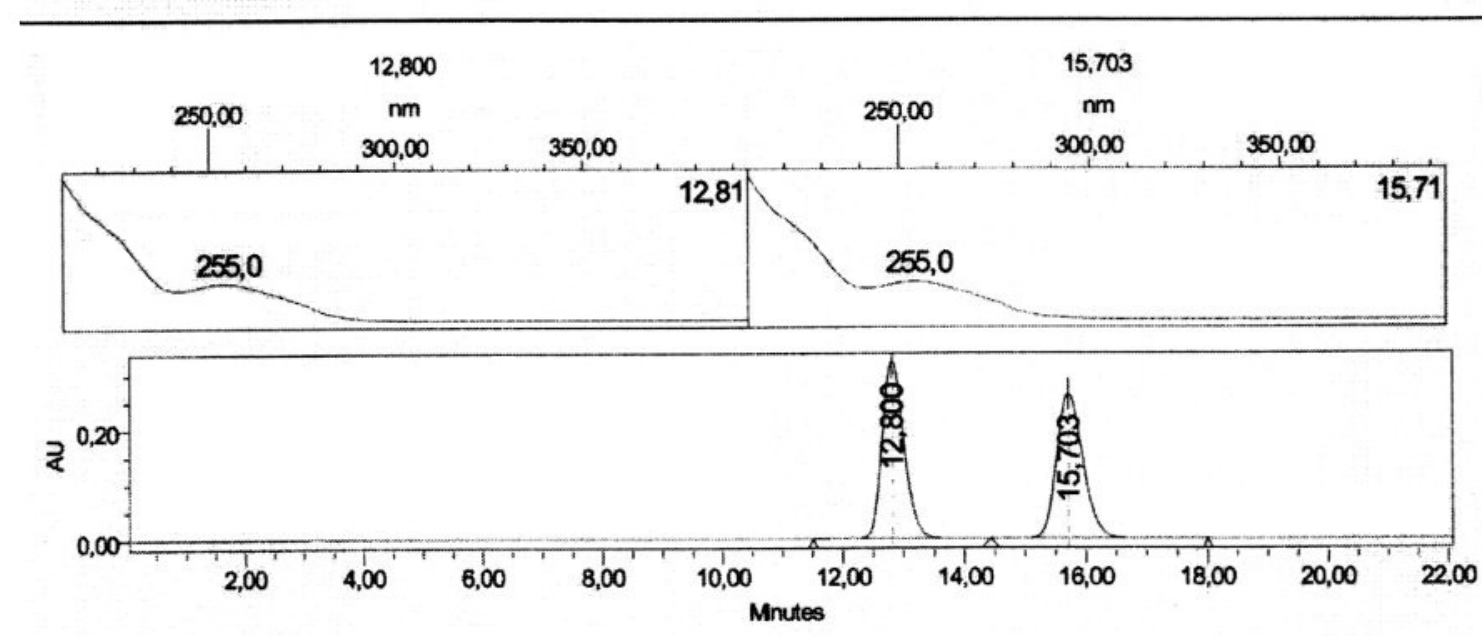

Colome: $\infty$

Hexane/isopropand : $90 / 10$

Fow. $1 \mathrm{~m} / \mathrm{min}$

Proc. Orrl. Descr.: PDA 254,0 m

\begin{tabular}{|c|c|c|c|c|c|}
\hline & RT & Area & Height & \% Area \\
\hline 1 & 12,800 & 7933523 & 321778 & 49,98 \\
\hline 2 & 15,703 & 7940460 & 261895 & 50,02 \\
\hline
\end{tabular}



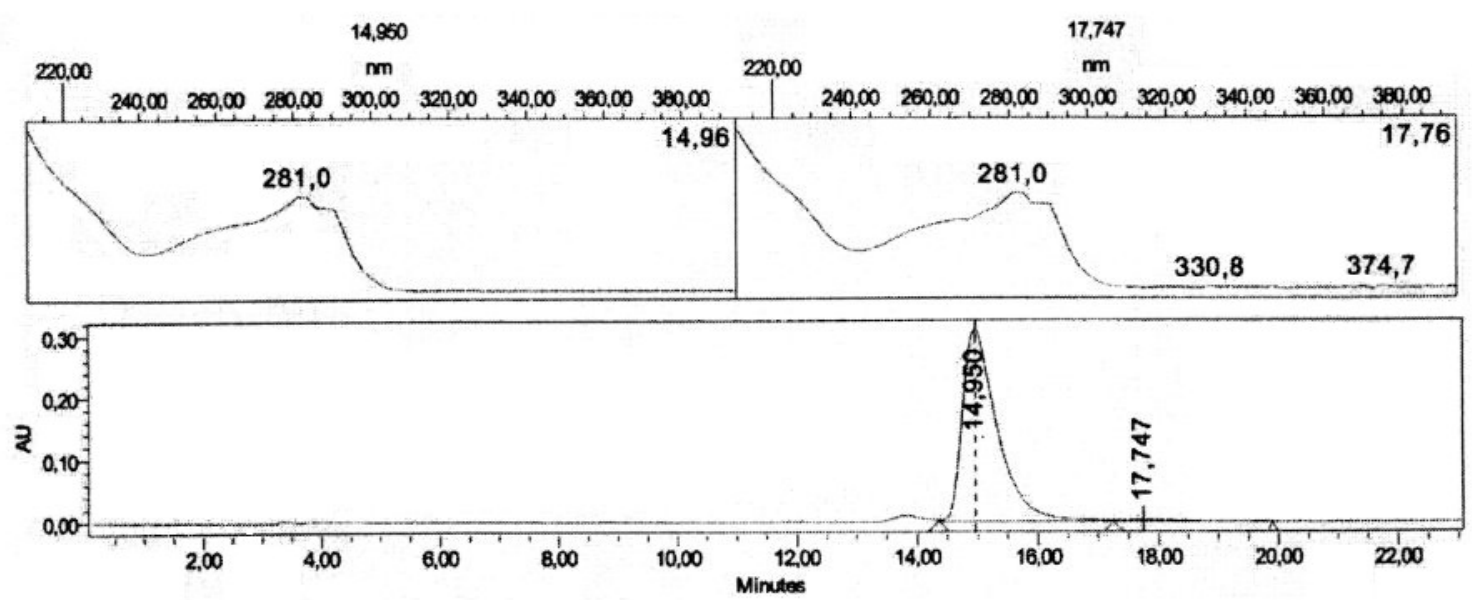

Colonne chirale: $O D$

Hexane/Ethanol: 94:6

Flow: $1 \mathrm{ml} / \mathrm{min}$

Processed Channel Descr. PDA 281,0 nm

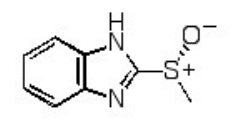

(entry 15)

Peak Results

\begin{tabular}{|r|c|c|r|}
\hline & RT & Area & \% Area \\
\hline 1 & 14,960 & 12311918 & 98,76 \\
\hline 2 & 17,747 & 154210 & 1,24 \\
\hline
\end{tabular}
300,00 350,00 350,00

16,91
250,00
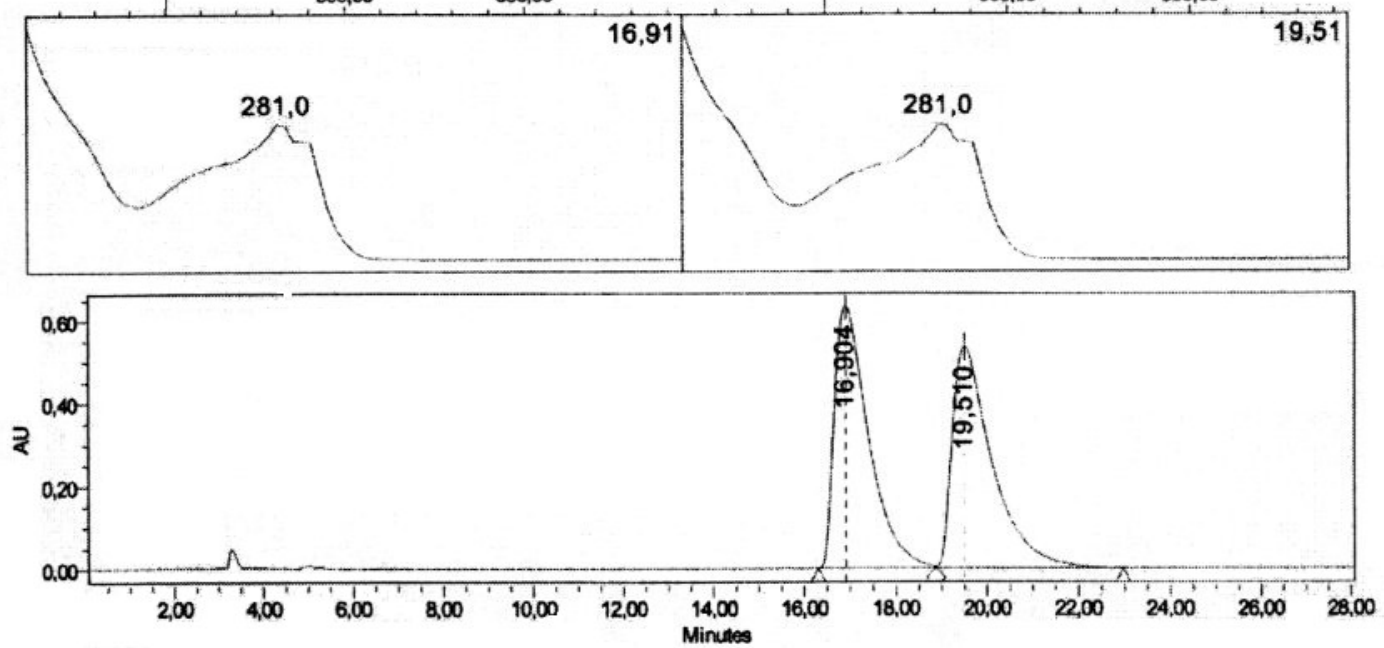

Colonne chirale: $O D$ Hexane/Ethanol: $94: 6$ Flow. $1 \mathrm{ml} / \mathrm{min}$ Processed Channel Descr. PDA 281,0 nm<smiles>C[S+]([O-])c1nc2ccccc2[nH]1</smiles>

(race mic)

Peak Results

\begin{tabular}{|l|l|c|c|c|}
\hline & Name & RT & Area & \% Area \\
\hline 1 & & 16,904 & 32546002 & 50,06 \\
\hline 2 & & 19,510 & 32464387 & 49,94 \\
\hline
\end{tabular}



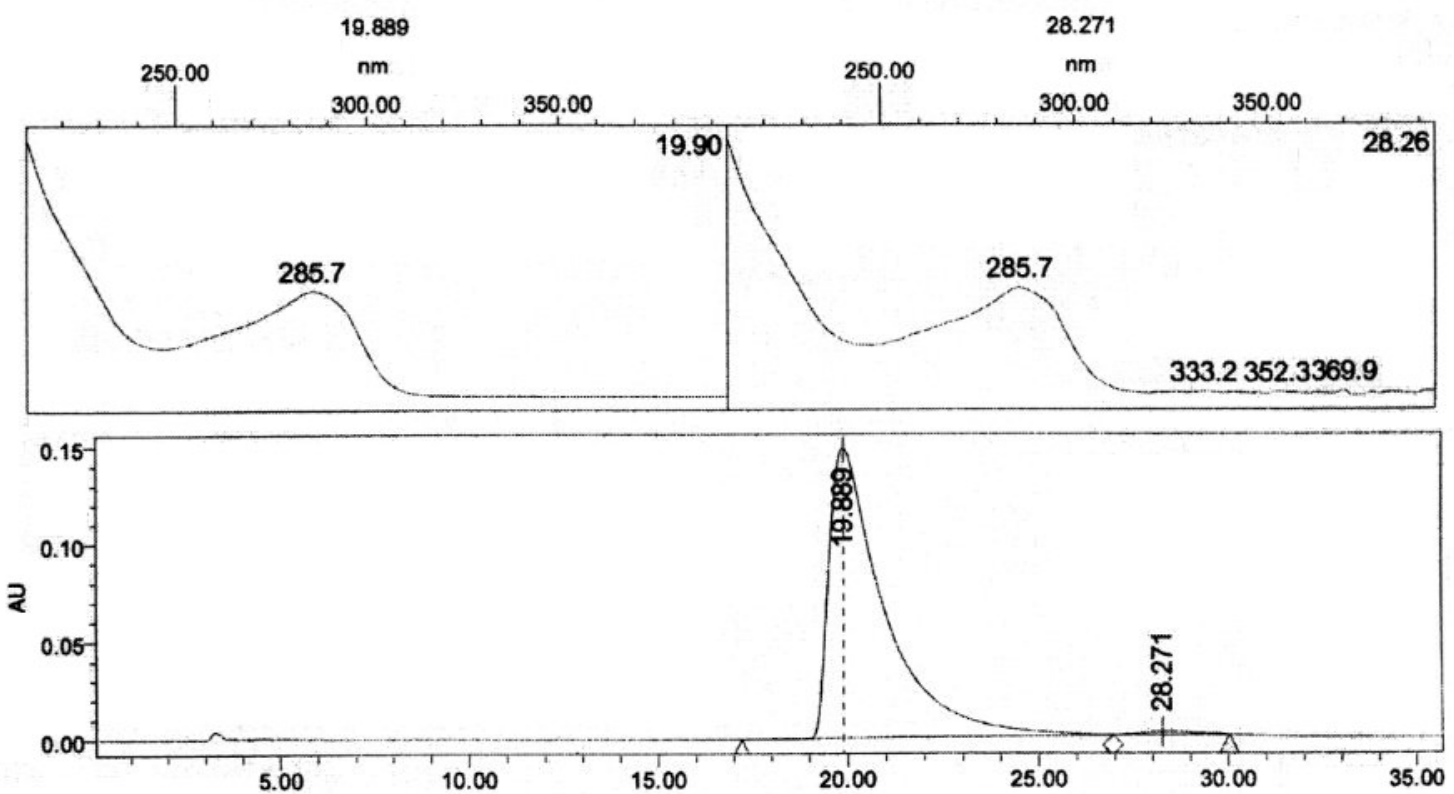

Processed Channel Descr. PDA 285.0 nn

Colonne chirale: $O D$

Hexane/lsopropanol: 80:20

Flow: $1 \mathrm{ml} / \mathrm{min}$
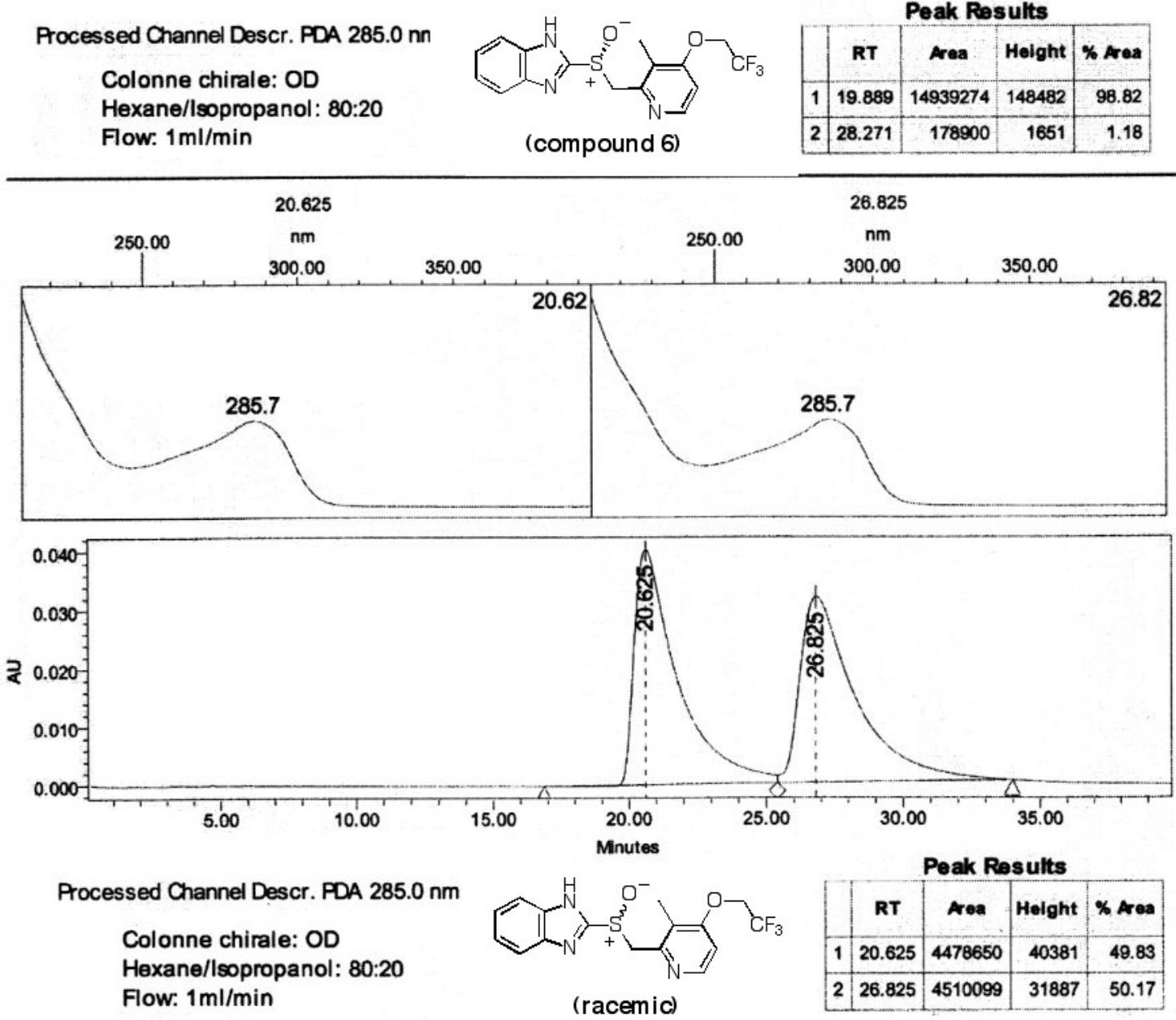